\title{
The star cluster-field star connection in nearby spiral galaxies
}

\section{Data analysis techniques and application to NGC 4395}

\author{
E. Silva-Villa and S. S. Larsen
}

\begin{abstract}
Astronomy Institute, University of Utrecht, Princetonplein 5, 3584 CC, Utrecht, The Netherlands e-mail: [e.silvavilla;s.s.larsen]@uu.nl
\end{abstract}

Received 16 February 2010 / Accepted 1 March 2010

ABSTRACT

\begin{abstract}
Context. It is generally assumed that a large fraction of stars are initially born in clusters. However, a large fraction of these disrupt on short timescales and the stars end up belonging to the field. Understanding this process is of paramount importance if we wish to constrain the star formation histories of external galaxies using star clusters.

Aims. We attempt to understand the relation between field stars and star clusters by simultaneously studying both in a number of nearby galaxies.

Methods. As a pilot study, we present results for the late-type spiral NGC 4395 using HST/ACS and HST/WFPC2 images. Different detection criteria were used to distinguish point sources (star candidates) and extended objects (star cluster candidates). Using a synthetic CMD method, we estimated the star formation history. Using simple stellar population model fitting, we calculated the mass and age of the cluster candidates.

Results. The field star formation rate appears to have been roughly constant, or to have possibly increased by up to about a factor of two, for ages younger than $\sim 300 \mathrm{Myr}$ within the fields covered by our data. Our data do not allow us to constrain the star formation histories at older ages. We identify a small number of clusters in both fields. Neither massive $\left(>10^{5} M_{\odot}\right)$ clusters nor clusters with ages $\geq 1$ Gyr were found in the galaxy and we found few clusters older than $100 \mathrm{Myr}$.

Conclusions. Based on our direct comparison of field stars and clusters in NGC 4395, we estimate the ratio of star formation rate in clusters that survive for $10^{7}$ to $10^{8}$ years to the total star formation to be $\Gamma \sim 0.03$. We suggest that this relatively low $\Gamma$ value is caused by the low star formation rate of NGC 4395 .
\end{abstract}

Key words. galaxies: individual: NGC 4395 - galaxies: star clusters: general - galaxies: star formation - galaxies: photometry

\section{Introduction}

It is commonly assumed that most (if not all) stars are formed in clusters. Clusters, due to dynamical and stellar evolution, dissolve (Spitzer 1987) and the stars that belong to them become part of the field stellar population of the galaxy. To use clusters as effective tools to constrain the formation and evolution of galaxies, it is necessary to improve our understanding of what fraction of stars end up as members of bound clusters and in the field, respectively.

Both field stars and star clusters have been studied extensively in the Milky Way and nearby galaxies. In principle, field stars hold information about star formation histories over the entire Hubble time and can therefore provide important information about galaxy formation and evolution, e.g., Edvardsson et al. (1993). Main sequence stars are present at all ages, and observations of the main sequence turn-off can constrain epochs of stellar formation especially in dwarf galaxies where relatively distinct bursts are often observed (Mateo 1998). Other features of the color-magnitude diagram (CMD), such as the subgiant and horizontal branches, giant and asymptotic branches, red clump stars, and red and blue supergiants can provide information about specific epochs of star formation. However, it is necessary to apply a more sophisticated modeling of the CMD to reconstruct star formation histories, taking into account different effects, e.g., incompleteness, resolution, depth of the observations, extinction and chemical composition. Tosi et al. (1991) developed a method that takes these effects into account and attempts to use all the information available in a CMD to reconstruct the field star formation history of a galaxy. The synthetic CMD method creates a synthetic population that is compared to the observed CMD to constrain the star formation history (SFH) of the field stars in a galaxy. Many subsequent studies have refined this method (Dolphin 1997; Harris \& Zaritsky 2001; Skillman \& Gallart 2002). The SFHs of many galaxies in the Local Group and nearby have been studied using the synthetic CMD method: Brown et al. (2008) for M31, Harris \& Zaritsky (2009) for the LMC, Harris \& Zaritsky (2004) for the SMC, Barker et al. (2007) for the M33, Cole et al. (2007) for Leo A, Young et al. (2007) for Phoenix, Annibali et al. (2009) for NGC 1705, Williams et al. (2009) for M 81, Larsen et al. (2007) for NGC1313 and Rejkuba et al. (2004) for NGC 5128, among many others.

On the other hand, the study of cluster systems and disruption processes can provide important insight into the origin of field stars in a galaxy. Clusters disrupt by means of a variety of mechanisms, including "infant mortality", stellar evolution, two-body relaxation, and tidal shocks that have been extensively studied by Boutloukos \& Lamers (2003), Lada \& Lada (2003), Lamers et al. (2005), Baumgardt (2009), Fall (2006), Elmegreen (2008), Whitmore (2007), and Bastian \& Gieles (2008) among others. The analysis of star clusters is often based on a comparison between the observed spectral energy distribution and theoretical models, which provides information about the ages and masses of the studied clusters and, in turn, their dissolution. 
However, the comparison of models with observations also remains affected by many uncertainties, e.g., binarity, completeness effects.

Some studies have started to address the relation between field stars and clusters more explicitly. The number of stars that were formed in clusters has been estimated to be $70 \%-90 \%$ in the solar neighbourhood, while $50 \%-95 \%$ of these embedded clusters dissolve in a few Myr (Lada \& Lada 2003; Lamers \& Gieles 2008). Gieles \& Bastian (2008) estimated that only 2-4\% of the global star formation rate in the SMC happened in bound star clusters. It is currently unknown what fraction of stars are initially born in clusters in SMC, but if this fraction were as large as in the Solar neighbourhood this would imply that there is also a large infant mortality rate in the SMC. Bastian (2008) studied the relation between the cluster formation rate and the star formation rate $\left(\Gamma=\frac{\mathrm{CFR}}{\mathrm{SFR}}\right)$ using archival Hubble images for high star formation rate galaxies and additional galaxies/clusters from literature. He found this fraction to be $\Gamma \sim 0.08$ and thus concluded that the fraction of stars formed in (bound) clusters represents $8 \%$ of the total star formation. On the other hand, Gieles (2009) found that $\Gamma$ is given by $0.05 \leq \Gamma \leq 0.18$ in the galaxies M 74, M 101, and M51. In most of these cases, however, it is difficult to tell whether there is a genuine "field" mode of cluster formation, or whether all stars form initially in clusters of which a large fraction dissolves rapidly.

We aim to analyze the relation between field stars and star clusters in different environments and address the question of whether or not there is a constant cluster formation "efficiency". To this end, we use Hubble Space Telescope (HST) images of a set of five galaxies (NGC 4395, NGC 1313, NGC 45, NGC 5236, and NGC 7793), which are nearby, face-on, spirals that differ in their morphologies, star, and cluster formation histories. The cluster systems of these galaxies were studied by Mora et al. (2009) using the same Hubble images analyzed in this work. Mora et al. observed significant variations in the cluster age distributions of these galaxies. The galaxies are sufficiently nearby ( $\sim \mathrm{Mpc})$ for the brighter field stars to be well resolved in HST images, so that (recent) field star formation histories can be constrained by means of the synthetic CMD method. We can therefore take advantage of the superb spatial resolution of HST images to study field stars and clusters simultaneously within specific regions of these galaxies.

As a pilot work, this paper is devoted to presenting and testing all the analysis procedures, such as detection of stars and cluster candidates, completeness tests and photometry, and derivation of field star and cluster age distributions. We discuss our implementation of the synthetic CMD method as an IDL program and carry out tests of this program. To test our procedures we use the galaxy NGC 4395. The methods described in this paper will be used in our study of the rest of the galaxy sample (Silva-Villa et al. 2010, in prep.).

This article has the following structure. In Sect. 2, we provide general information about NGC 4395. We present the observations, data reduction, and photometry, where we differentiate the point sources (star candidates) from the extended objects (cluster candidates) in Sects. 3 and 4. Section 5 is devoted to the analysis of the star and cluster properties. Finally, in Sect. 6 we present the discussion and conclusions.

\section{NGC 4395}

According to the NASA/IPAC Extragalactic Database (NED), NGC 4395 is a late-type spiral classified as type SA(s)m. It harbours the closest and least luminous known example of a Seyfert 1 nucleus (Filippenko \& Sargent 1989). Following Larsen \& Richtler (1999), we adopt a distance modulus of $(m-M)=28.1(D \approx 4.2 \mathrm{Mpc})$ and an absolute magnitude $M_{B}=-17.47$, intermediate between the Small and Large Magellanic Cloud. We assume a Galactic foreground extinction for NGC 4395 of $A_{B}=0.074$ (Schlegel et al. 1998).

The cluster system of NGC 4395 was first studied by Larsen \& Richtler (1999), using ground-based imaging. Using multiband $(U B V R I H \alpha)$ photometry, Larsen \& Richtler identified 2 young clusters in this galaxy, although their observations were limited to objects with $M_{V} \leq-8.5$. In their work, NGC 4395 is part of a sample of 21 galaxies. Compared to the remaining sample, NGC 4395 exhibits an exceptionally small number of star clusters and a low specific luminosity (ratio of cluster- to total galaxy light; Larsen \& Richtler 2000). Using HST images, Mora et al. (2009) could detect clusters based on their sizes and found a total of 44 clusters in NGC 4395 to a magnitude limit of $M_{B}=-3.3$. Compared to the other 4 galaxies in their sample, Mora et al. reached the same conclusions as Larsen \& Richtler, i.e., NGC 4395 has a small number of star clusters. Mora et al. estimated the ages and masses of the clusters detected, showing that the star cluster system contains no objects with ages older than $10^{9} \mathrm{yr}$ and includes clusters with masses ranging from 200 to $\sim 3 \times 10^{4} M_{\odot}$.

Until now, no study of resolved field stars has been performed in this galaxy. However, Larsen \& Richtler (2000) found that NGC 4395 has one of the lowest area-normalised star formation rates of all objects in their sample.

\section{Observation and data reduction}

Images of NGC 4395 were taken using the Wide Field Channel on the Advanced Camera for Surveys (ACS/WFC) and the Wide Field Planetary Camera 2 (WFPC2), both on board the Hubble Space Telescope. The resolution of the detectors are $0 . ' 05,0.046$, and 0.' 1 per pixel for ACS/WFC, WFPC2/PC, and WFPC2/WF, respectively. At the distance of NGC 4395, 0'05 corresponds to a linear scale of $\sim 1 \mathrm{pc}$.

Two different fields of the galaxy were observed, covering the two spiral arms (see Fig. 1). The images were taken using the filters $F 336 W(\sim U)$ using $W F P C 2$ and $F 435 W(\sim B), F 555 W$ $(\sim V)$ and $F 814 W(\sim I)$ using ACS, for each field. Each exposure was divided into two sub-exposures to eliminate cosmic-ray hits. For the ACS images, these sub-exposures were also dithered to allow removal of cosmetic defects. Table 1 summarizes the observations.

The data were processed with the standard STScI pipeline. The raw ACS images were drizzled using the multidrizzle task (Koekemoer et al. 2002) in the STSDAS package of IRAF ${ }^{1}$. The default parameters were used, but automatic sky subtraction was disabled. The WFPC2 images were combined and corrected for cosmic rays using the crrej task with the default parameters.

\subsection{Object detection}

At the distance of NGC 4395, the spatial resolution of the HST images allows us to distinguish field stars (point sources with typical $F W H M \sim 2$ pixels) and star clusters (extended sources with typically $F W H M \sim 3$ pixels or greater) in the images. We

\footnotetext{
1 IRAF is distributed by the National Optical Astronomical Observatory (NOAO), which is operated by the Association of Universities for Research in Astronomy, Inc, under cooperative agreement with the National Science Foundation
} 
E. Silva-Villa and S. S. Larsen: The star cluster-field star connection in nearby spiral galaxies

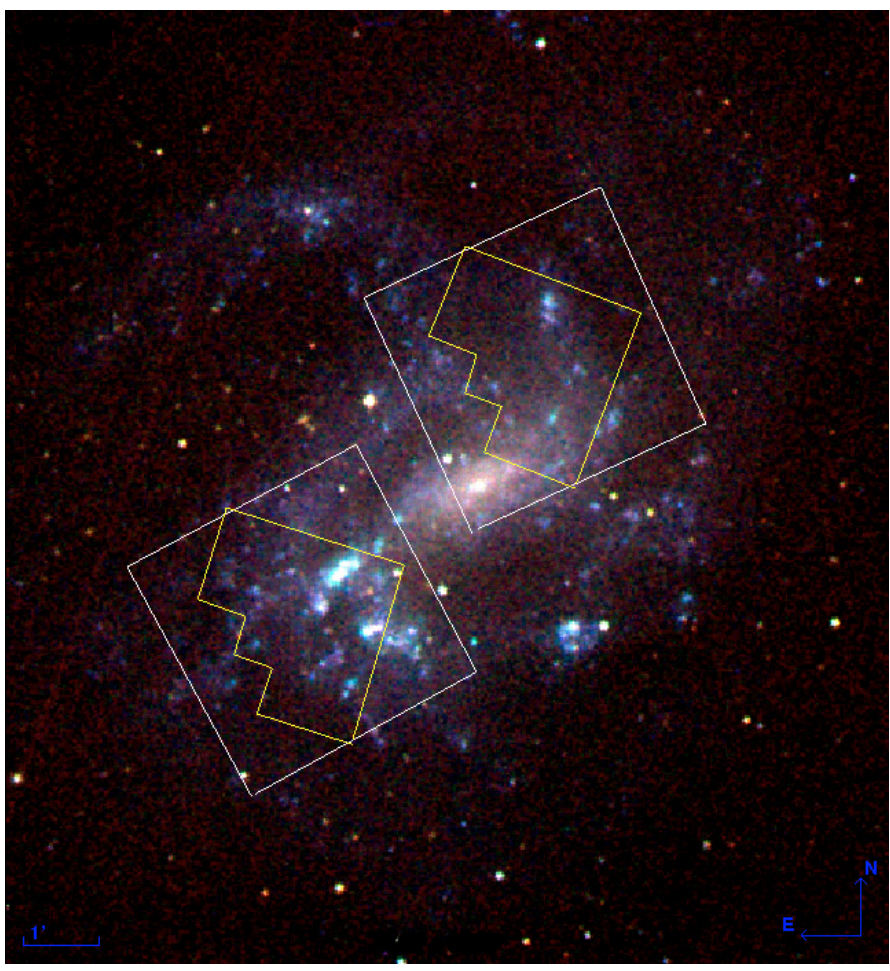

Fig. 1. Second Palomar Sky Survey images of NGC 4395 combined using Aladin software. HST/ACS (white lines) and HST/WFPC2 (yellow lines) fields covered by our observations are indicated.

analyzed the two separately and applied different detection criteria optimised for each type of object:

\section{- Field star candidates (point sources)}

To detect field stars, we created an averaged image (using the bands $B, V$, and $I$ ) for each field. We ran the daofind task in IRAF for the detection of stars using a $4 \sigma$ detection threshold and a background standard deviation of $\sim 0.02$ in units of counts per second.

\section{- Star cluster candidates (extended sources)}

Cluster detection was also performed on the averaged image. The object detection was performed using SExtractor V2.5.0 (Bertin \& Arnouts 1996). The parameters used as input for this program were 6 connected pixels, all of them with $10 \sigma$ over the background, to remove point- and spurious sources as much as possible. From the output file of SExtractor, we kept the coordinates of the objects detected and the FWHM calculated by this program. The coordinates found with SExtractor were then passed to ishape in BAOLab (Larsen 1999). Ishape models star clusters as analytical King (1962) profiles (with concentration parameter $\left.t_{\text {tidal }} / r_{\text {core }}=30\right)$ and takes the instrument's PSF, created over the average image during the photometry, into account (see Sect. 4 for details on the creation of the PSF). By minimization of a $\chi^{2}$-like function, ishape calculates the best-fit cluster coordinates, size, i.e., FWHM, signal-to-noise ratio, and the $\chi^{2}$ of the best fit. From the output of ishape we saved the coordinate of the objects, the FWHM, the $\chi^{2}$ of the fit, and the signal-to-noise ratio (calculated within the fitting radius of 4 pixels).

For each WFPC2 chip, between five and seven common stars were visually selected and used to convert the coordinate list from ACS to WFPC2 frames using the task geomap in IRAF. The transformations had an rms of $\sim 0.15$ pixels.

\section{Photometry}

The photometry of NGC 4395 was performed following standard aperture and PSF fitting photometry procedures as described in the following.

\subsection{Field stars}

Because of the crowding in our fields, we performed PSF fitting photometry to study the field stars. A set of bona fide stars were selected by eye to construct the PSF (for each band a different group of stars were used because the same stars might have different brightnesses in different bands). The PSF photometry was performed with DAOPHOT in IRAF. We selected the PSF stars by measuring the $F W H M$ (using imexamine) and selecting point sources smaller than $F W H M \approx 2.2$ pixels. As far as possible, we tried to include isolated stars distributed over the whole image.

The raw magnitudes were converted to the Vega magnitude system using the HST zero-points taken from HST webpages $^{2}$ after applying aperture corrections to a nominal 0.5 aperture (see Sect. 4.4 for a more detailed description of how aperture corrections were determined). The zero-points used were $Z P_{B}=25.767, Z P_{V}=25.727$, and $Z P_{I}=25.520 \mathrm{mag}$.

Figure 2 shows the errors in our PSF photometry ( $1 \sigma$ error) versus magnitude. The errors increase strongly below magnitudes of $\sim 26$ in each band, corresponding to absolute limits of $\sim-2$ mag at the distance of NGC 4395.

A total of $\sim 30000$ stars were found in each field. A combined Hess diagram for both fields is shown in Fig. 3 (a Hess diagram plots the relative frequency of stars at different colormagnitude positions). Various phases of stellar evolution can be recognised in the Hess diagram:

1. Main sequence and possible blue He-core burning stars at $V-I \sim 0$ and $-2 \leq V \leq-8$

2. Red He core burning stars at $1.2 \leq V-I \leq 2.5$ and $-2.5 \leq$ $V \leq-6.5$;

3. RGB/AGB stars at $1 \leq V-I \leq 3$ and $-0.5 \leq V \leq-2.5$.

Overplotted in Fig. 3 are theoretical isochrones from the Padova group (Marigo et al. 2008) for five different ages using metallicity $Z=0.008$ (red lines). The $\mathrm{O}$ abundance of HII regions in NGC 4395 was measured by Roy et al. (1996) to be $12+\log \mathrm{O} / \mathrm{H}$ $=8.33 \pm 0.25$, or about $1 / 3$ solar (Grevesse \& Sauval 1998), suggesting an overall metallicity similar to that of the LMC. This is consistent with our analysis in Sect. 5, which shows that isochrones of LMC metallicity reproduce our data more closely. The white line indicates the $50 \%$ completeness limit (see Sect. 4.3).

\subsection{Star clusters}

Our detection criteria is met by 16463 objects, i.e., 6 connected pixels with $10 \sigma$ above the local background level. For the clusters, we carried out aperture photometry. An aperture radius of 6 pixels was used for the ACS images. At the distance of NGC 4395, 1 ACS/WFC pixel corresponds to $\sim 1 \mathrm{pc}$, hence the chosen source aperture contains about 2 half-light radii for a typical star cluster. Our sky annulus had an inner radius of 8 pixels and a width of 5 pixels. For the WFPC 2 images, apertures covering the same area were used (source aperture $=3$ pixel radius, sky annulus $=4$ pixel inner radius and 2.5 pixels width).

\footnotetext{
2 http://WWW.stsci.edu/hst/acs/analysis/zeropoints/\# tablestart
} 
A\&A 516, A10 (2010)

Table 1. Journal of HST/ACS and HST/WFPC2 observations for both fields (F1 and F2) in NGC 4395.

\begin{tabular}{|c|c|c|c|}
\hline Proposal ID & Date & Filter & Total exp. time (s) \\
\hline \multicolumn{4}{|c|}{ NGC $4395(\mathrm{~J} 2000.0) \alpha: 12^{\mathrm{h}} 26^{\mathrm{m}} 00^{\mathrm{s}} \delta:+33^{\circ} 31^{\prime} 04^{\prime \prime}$} \\
\hline 9774 & 2004 Jun. 12 & $F 435 W(B)$ & 680 \\
\hline 9774 & 2004 Jun. 12 & $F 555 W(V)$ & 680 \\
\hline 9774 & 2004 Jun. 12 & $F 814 W(I)$ & 430 \\
\hline \multicolumn{4}{|c|}{ NGC $4395(\mathrm{~J} 2000.0) \alpha: 12^{\mathrm{h}} 26^{\mathrm{m}} 00^{\mathrm{s}} \cdot 50 \delta:+33^{\circ} 30^{\prime} 58^{\prime \prime \prime} 3$} \\
\hline 9774 & 2004 Jun. 12 & $F 336 W(U)$ & 2400 \\
\hline \multicolumn{4}{|c|}{ NGC $4395(\mathrm{~J} 2000.0) \alpha: 12^{\mathrm{h}} 25^{\mathrm{m}} 45^{\mathrm{s}} \cdot 20 \delta:+33^{\circ} 34^{\prime} 28^{\prime \prime}$} \\
\hline 9774 & 2004 Jun. 18 & $F 435 W(B)$ & 680 \\
\hline 9774 & 2004 Jun. 18 & $F 555 W(V)$ & 680 \\
\hline 9774 & 2004 Jun. 18 & $F 814 W(I)$ & 430 \\
\hline \multicolumn{4}{|c|}{ NGC $4395(\mathrm{~J} 2000.0) \alpha: 12^{\mathrm{h}} 25^{\mathrm{m}} 42^{\mathrm{s}} \cdot 72 \delta:+33^{\mathrm{o}} 34^{\prime} 22^{\prime \prime} 6$} \\
\hline 9774 & 2004 Jun. 18 & $F 336 W(U)$ & 2400 \\
\hline
\end{tabular}

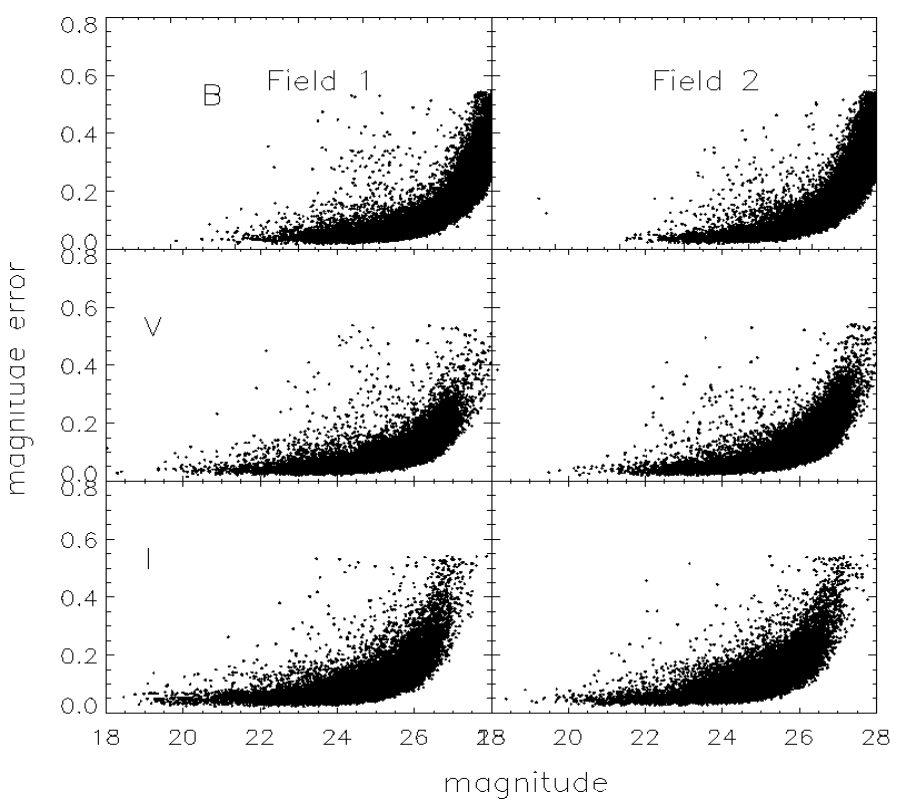

Fig. 2. Magnitude errors for the stars detected using PSF fitting photometry over the two fields for the bands $B, V$, and $I$.

Of the 16463 objects detected with SExtractor/ishape, a total of 4472 candidates have measured four band photometry.

ACS magnitudes were converted to vega magnitude system using the same tables as for the field stars. WFPC2 magnitudes were converted to the Vega magnitude system using the zeropoints taken from the webpages ${ }^{3}$ of HST. Charge transfer efficiency (CTE) corrections were applied following the equations from Dolphin $(2000)^{4}$.

To select star cluster candidates, we used 3 criteria:

\section{- Size:}

We measured the sizes of the objects. Figure 4 shows the FWHM distributions for SExtractor and ishape. The SExtractor histogram peaks at $\sim 2.2$ pixels, corresponding to the stellar PSF. The ishape histogram peaks at 0 pixels, as ishape takes the PSF directly into account. Based on the FWHM distributions, we decided to use a criteria of $F W H M_{\text {SExtractor }} \geq 2.7$ pixels and $F W H M_{\text {ishape }} \geq 0.7$ pixels as a first selection of extended sources. These two limits

\footnotetext{
3 www.stsci.edu/instruments/wfpc2/Wfpc2_ch52\#1933986

${ }^{4}$ Last update May 12, 2008:

http://purcell.as.arizona.edu/wfpc2_calib/
}

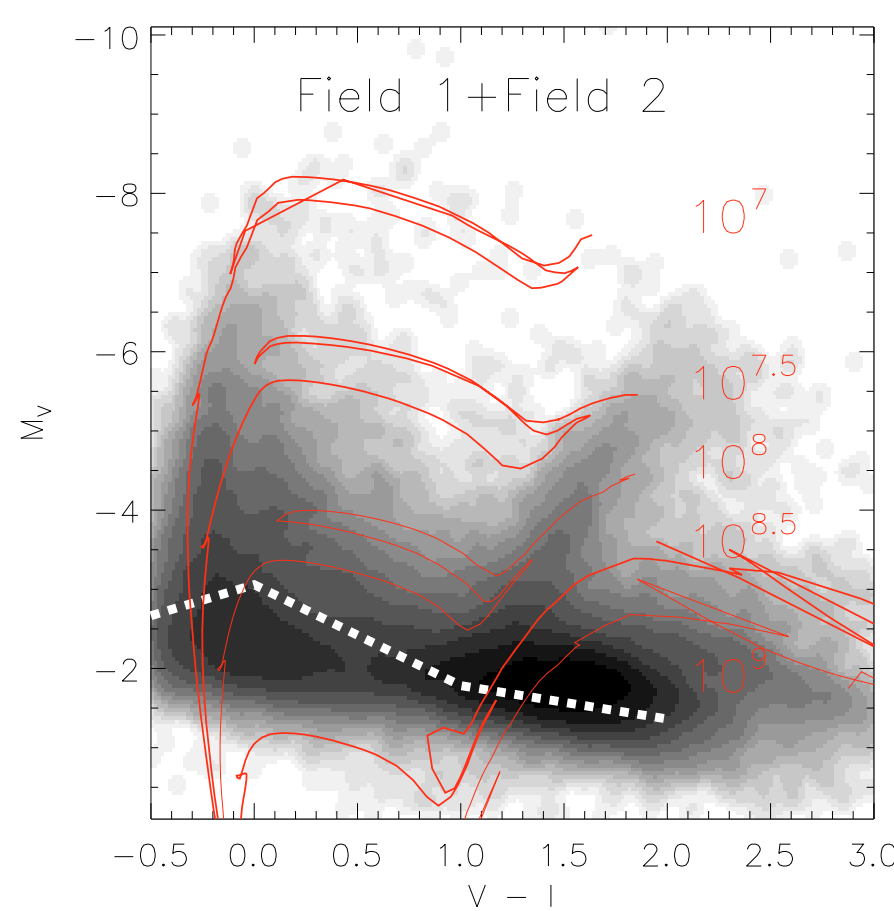

Fig. 3. Hess diagram for the field stars in both fields. The dashed white line represents the $50 \%$ completeness for the first field. Red lines are Padova 2008 theoretical isochrones for the ages $10^{7}, 10^{7.5}, 10^{8}, 10^{8.5}$, and $10^{9} \mathrm{yr}$ using LMC metallicity.

correspond to a physical cluster half-light radius of $\sim 1 \mathrm{pc}$ or greater at the distance of NGC 4395. Many of the candidate clusters we detect are low-mass and have irregular profiles often dominated by a few stars, so we chose to rely on both SExtractor and Ishape size measurements to achieve a more robust rejection of unresolved sources.

- Magnitude:

Mora et al. (2009) ran a completeness analysis of artificial star clusters of different $F W H M$ s in five nearby galaxies, including NGC 4395. The results presented in their work establish a $50 \%$ limit between $25 \leq m_{B} \leq 26$ mag for objects with $F W H M=[0.1$ (point sources) , 1.8] (see Mora et al. (2007, 2009) and Sect. 4.3 for more details).

By performing four band photometry, we set a magnitude cut-off at $m_{V} \leq 23$, which represents objects brighter than $M_{V} \sim-5$ at the distance of the galaxy. This limit is brighter than that found by Mora et al. (2007) by $\sim 2 \mathrm{mag}$. 


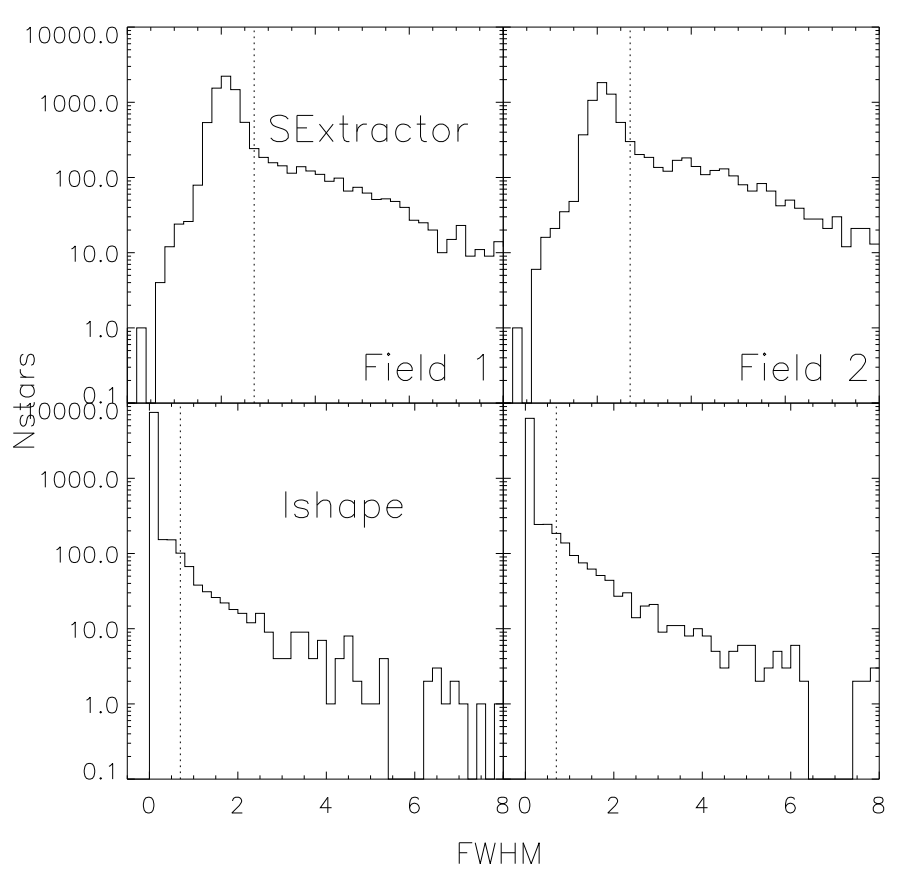

Fig. 4. Histograms of the FWHM of the objects detected with SExtractor (top panel) and ishape (bottom panel). The vertical dotted lines represent the limits used to select the extended objects $\left(F W H M_{\text {SExtractor }} \geq 2.7\right.$ and $F W H M_{\text {ishape }} \geq 0.7$ pixels $)$

The main difference between the selection criteria of Mora et al. (2007) and this work is in the size limits of ishape, of half of a pixel (Mora et al. used $F W H M_{\text {ishape }} \geq 0.2$ ), hence we expect our data not to be significantly affected by incompleteness to our magnitude cutoff. However, even at our limit of $M_{V}=-5$, there is a risk that a few stars may dominate the light originating in a cluster, making it difficult to differentiate a real cluster from a couple of stars that, by chance, could be in the same line of sight.

- Color:

Without taking into account any significant reddening, all clusters, including globulars, will have colors bluer than $V-I \sim 1.5$, e.g., Forbes et al. (1997), Larsen et al. (2001). We therefore make a color cut at $V-I=1.5$.

We wish to emphasise that there is probably no unique combination of criteria that will lead to the detection of all bona-fide clusters in the image and at the same time produce no false detections. At low masses and young ages in particular, the light profiles may be dominated by individual bright stars. Out of 4472 candidate objects, a total of 22 objects fulfill the three criteria stated above and will be considerate in the remain of this study to be star clusters. These 22 clusters were visually inspected in our images to determine whether they resemble a cluster. An example of the clusters detected is presented in Fig. 5. We investigated the large number of rejected objects and found that they were rejected for many reasons: (1.) the area covered by the two detectors differs by a factor of $\sim 2 ;$ (2.) many of the objects are too faint in the $U$ band; (3.) the magnitude cut-off removes many objects, e.g., from a total of 4475 detected objects, only 1351 satisfy the magnitude limit, leading to the loss of $\sim 70 \%$ detections; and (4.) a large fraction of the objects have $F W H M$ s smaller than our limits.

For the extended objects, a two-color diagram, based on the photometry in all of the available 4 passbands, is presented in Fig. 6. Clusters in both fields are depicted with their
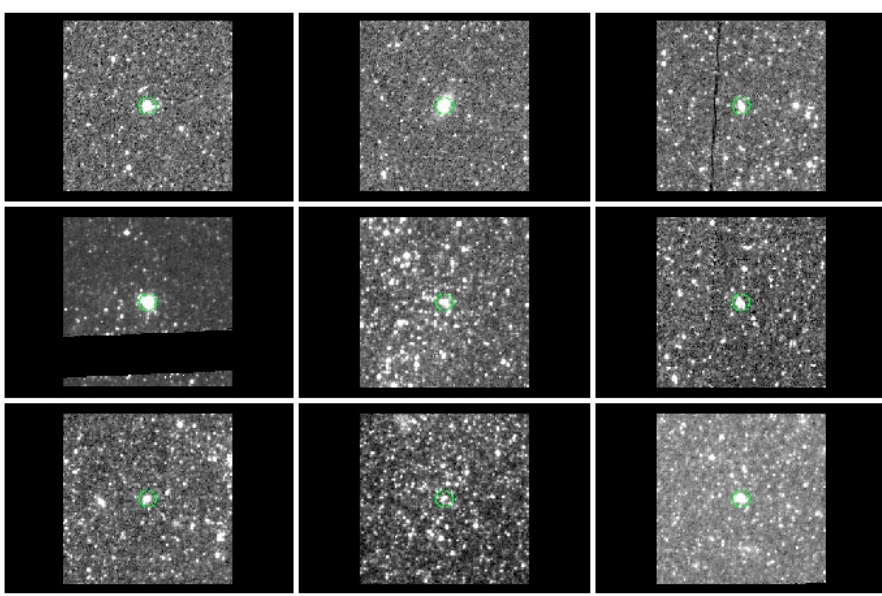

Fig. 5. Example stamps of the clusters that satisfy the criteria stated in this work. Each cluster image has a dimension of $100 \times 100$ pixels $(\sim 100 \times 100 \mathrm{pc})$.

respective photometric errors and corrected for foreground extinction $\left(A_{B}=0.074\right)$. Using GALEV models (Anders \& Fritze-v. Alvensleben 2003), Padova isochrones (Bertelli et al. 1994), a Salpeter's IMF (Salpeter 1955), and LMC (dashed line) or solar (dash-dotted line) metallicities, we overplotted the track that a cluster follows from ages between $6.6 \leq \log (\tau / \mathrm{yr}) \leq 10.2$ (each $\Delta \log (\tau)=0.5$ dex age in log units being indicated). We observe considerable scatter in the two-color diagram. For these relatively low-mass clusters, the discreteness of the initial mass function will be important and might contribute significantly to the scatter (Girardi et al. 1995; Cerviño \& Luridiana 2006; Maíz Apellániz 2009). A more detailed study of stochastic effects will be presented in a forthcoming paper (Silva-Villa et al. 2010, in prep.) This scatter was previously observed by Mora et al. (2009) (see their Fig. 6). Considering this scatter, it is clear that ages derived by a comparing observed and model colors should be treated with some caution.

\subsection{Completeness test}

To determine the completeness limits of our field-star photometry, we generated synthetic images with artificial stars. From 20 to $28 \mathrm{mag}$, in steps of $0.5 \mathrm{mag}$, five images per step were created and analyzed with exactly the same parameters used in the original photometry. For each combination of color and magnitude, 528 artificial stars were added using 4 different regions over the image to cover crowded and uncrowded areas. These test images were created using the task mksynth in BAOLab (Larsen 1999) and using the original PSF images created during the photometry procedures (see Sect. 4.1). The separation between two consecutive stars was 100 pixels (without any sub-pixel variations), avoiding possible overlaping among the stars. The resulting test images were added to the science images using the task imarith in IRAF. As an example, a subsection of an image, both test and science, is presented in Fig. 7, where the fake stars have magnitudes $m_{V}=21$.

To performe a realistic completeness analysis it is in principle necessary to sample the three-dimensional $(B, V, I)$ color space. However, since different colors are tightly correlated with each other, the problem can be reduced to a two-dimensional one. Figure 8 was generated using Padova 2008 isochrones (Marigo et al. 2008), assuming solar metallicity, in the color range from -1 to 2 , for $B-V$ and $V-I$ and shows that there 


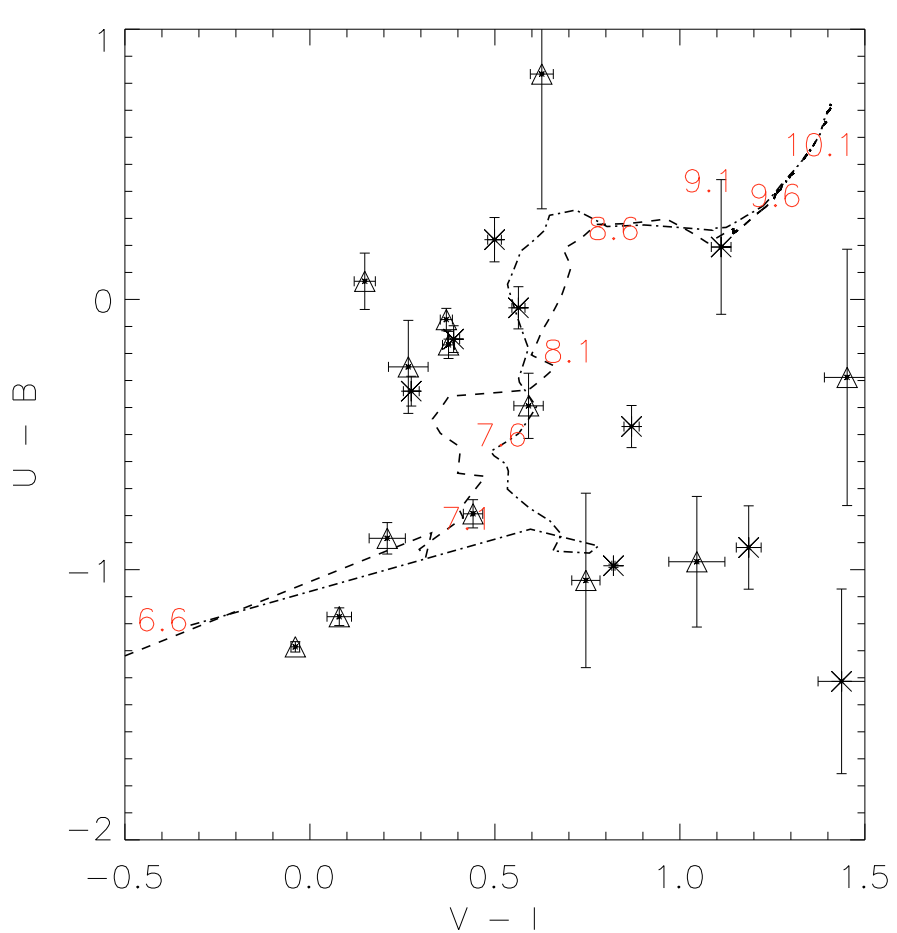

Fig. 6. Two-color diagram for the star clusters detected in both fields, $\times$ represents the first field and $\Delta$ the second field. The lines represent the theoretical GALEV track for a cluster with LMC (dashed line) or solar (dash-dotted line) metallicity, adopting Padova isochrones, a Salpeter IMF, and ages between $6.6 \leq \log (\tau / \mathrm{yr}) \leq 10.1$.
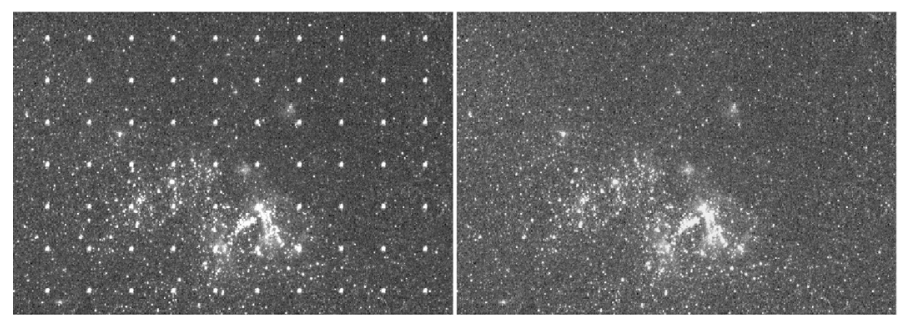

Fig. 7. Subsection of the original image (right) and completeness image (left).

is a nearly $1: 1$ relation between these two colors (the red line in Fig. 8 represents a 1:1 relation, but not an accurate fit to the data). We created the images for the completeness test described above using this approximation between the three bands, i.e., if a $B$ image has stars with $m_{B}=21$ and $B-V=1$, then the $V$ and $I$ images will have stars of $m_{V}=20$ and $m_{I}=19$, respectively, allowing us to perform a study of magnitudes and color variations over the images.

Based on these tests, we found an average of $50 \%$ completeness limits for the whole color range at $m_{B}=26.69, m_{V}=26.55$, and $m_{I}=26.42$ for the first field and $m_{B}=26.71, m_{V}=26.49$, and $m_{I}=26.39$ for the second field. Figure 9 shows the completeness diagram obtained from this analysis for the first field (for the second field, the figure is similar) and each magnitude.

The color-dependent 50\% completeness limit is shown as a white dashed line in the Hess diagram (see Fig. 3). Since the completeness functions are very similar for the two fields, we can combine the photometry for both fields and use just one set of completeness tests in the following analysis.

We did not performe a completeness test for star clusters but refer to the tests performed by Mora et al. (2007, 2009),

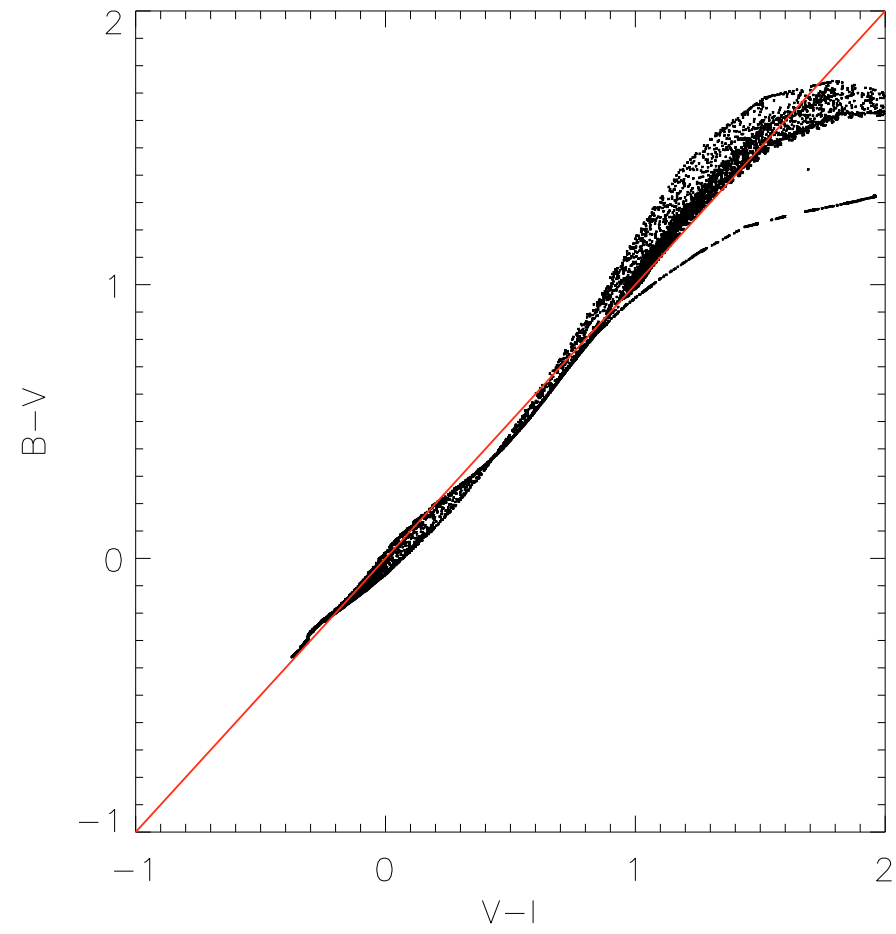

Fig. 8. Two color diagram for theoretical values of $B, V$ and $I$ bands from Padova 2008 isochrones adopting LMC metallicity for ages between $10^{6.6}$ to $10^{10} \mathrm{yr}$. The red line represents a $1: 1$ relation between the colors $B-V$ and $V-I$.

who used the same data and very similar cluster detection procedures. These authors created artificial star clusters using different $F W H M$ s from 0.1 pixels (stars) to 1.8 pixels and a range of magnitudes from 16 to 26 for three square grids in different positions over the image, trying to cover crowded and non-crowded areas. To create the fake clusters, they assumed a King (1962) profile with $r_{\text {tidal }} / r_{\text {core }}=30$. Using the $m k c m p p s f$ task on BAOLab, fake extended objects were added to an empty image and then added to the science image to performe measurements. The selection criteria in Mora et al. $(2007,2009)$ is a $F W H M \geq(2.7,0.2)$ pixels for SExtractor and ishape, respectively. For high background levels, Mora et al. found a shallower detection limit; nevertheless, all the limits correspond a 50\% completeness limit between $m_{B} \approx 25$ and $m_{B} \approx 26,2-3$ magnitudes fainter than the cut at $V=23$ that we apply for the selection of cluster candidates.

\subsection{Aperture corrections}

The aperture corrections were determined separately for field stars and star clusters. The aperture corrections for the field stars were derived following standard procedures, while for extended objects we adopted the relations found by Mora et al. (2009).

- Field stars:

By "aperture corrections", we here mean corrections from the PSF-fitted instrumental magnitudes to aperture photometry for nominal radii of 0.5 . These were measured using a set of isolated visually selected stars across the images. From 0.'5 to infinity, we applied the Sirianni et al. (2005) values. The corrections obtained are of the order $\sim 0.1 \mathrm{mag}$ (see Table 2).

- Star clusters:

Mora et al. (2009) estimated a relation between the aperture corrections and the sizes ( $F W H M)$ of star clusters using the 


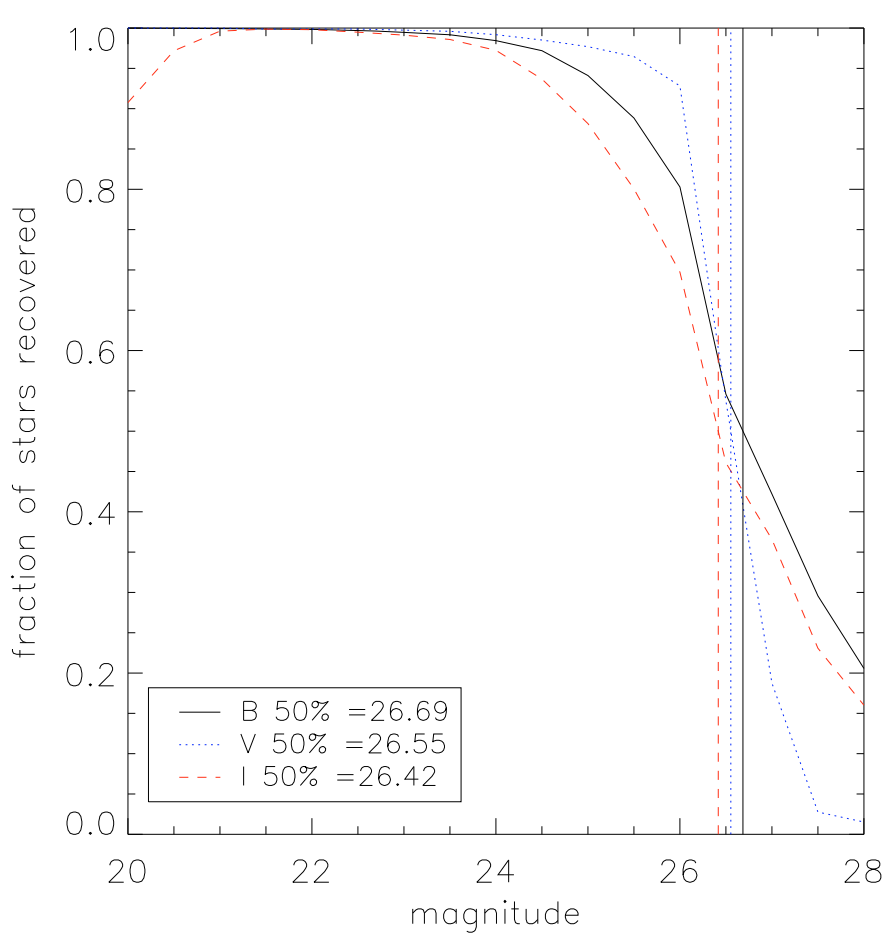

Fig. 9. Completeness diagrams for the bands $B, V$, and $I$ studied over the first field. Vertical lines represent the $50 \%$ completeness.

Table 2. For point source. $B, V$, and $I$ aperture corrections to a nominal aperture of $0 . ' 5$, estimated in this study.

\begin{tabular}{lccc}
\hline \hline & $\begin{array}{c}B_{F 435 W} \\
{[\mathrm{mag}]}\end{array}$ & $\begin{array}{c}V_{F 555 W} \\
{[\mathrm{mag}]}\end{array}$ & $\begin{array}{c}I_{F 814 W} \\
{[\mathrm{mag}]}\end{array}$ \\
\hline Field 1 & 0.07 & 0.03 & 0.08 \\
Field 2 & 0.05 & 0.08 & 0.06 \\
\hline
\end{tabular}

same data set used in this paper. Photometric parameters in both Mora et al. (2009, and ours) work are the same, allowing us to assume the relations found in their work and apply these aperture corrections (size-dependent) to our data, following Eq. (1) in Mora et al. (2009). This set of equations is also band-dependent, although we used the sizes of the objects measured on an average image.

The aperture corrections by Mora et al. (2009) correspond to a nominal aperture of 1'. 45 . From this nominal aperture to infinity, we adopted the values presented by Sirianni et al. (2005), although these corrections are $\sim 0.03 \mathrm{mag}$ for the bands $B, V$, and $I$ (within 1 '. 5 about $97 \%$ of the total energy is encircled).

\section{Star formation histories, ages, and masses}

Our main goal in this paper is to compare the field stars with the star cluster populations in NGC 4395. In the following section, we describe how we derive the star formation histories (SFHs) of the field stars and the ages and masses of the clusters. We then proceed to compare the two.

\subsection{Deriving star formation histories: approach and its testing}

To estimate the SFH of the field stars, we have used the synthetic CMD method. The synthetic CMD method consists of creating an artificial photometric distribution of stars taking into account photometric errors, distance modulus, IMF, binarity, and interstellar extinction. The relative weights of the stellar isochrones used in generating the synthetic CMD are adjusted until the closest possible match of the synthetic CMD to the observed one is obtained. These weights can then be translated to a SFH.

Following the work of Tosi et al. (1991), Dolphin (1997), and Aparicio et al. (1997), many authors have implemented this technique. Some examples are STARFISH by Harris \& Zaritsky (2002), IAC-Star by Aparicio \& Gallart (2004), IACpop by Aparicio \& Hidalgo (2009), among others. Skillman \& Gallart (2002) presented the results of a workshop (The Coimbra Experiment) devoted to comparing different methods and interpretations of the results from various groups using a homogeneous data set and physical inputs. The results showed a large scatter at young ages, but good agreement at old ages.

We implemented the synthetic CMD technique ourselves as an IDL program. We chose to develop our own code to include any features that we might wish (or need), and there is no clear preference in the literature for any of the other codes. The IDL code used in this work was based on our previous experience with the synthetic CMD technique (Larsen et al. 2002). The program searches for the best-fit synthetic Hess diagram using a maximum-likelihood technique. We assume that the likelihood of observing $k_{i}$ stars in the $i$ th pixel of the Hess diagram is given by the Poisson distribution $\mathcal{P}\left(k_{i} ; \mu_{i}\right)$, where $\mu_{i}$ is the number of stars expected in this pixel according to a given model Hess diagram. The program then searches for the linear combination of input isochrones that maximises the total likelihood $\mathcal{L}$ over the entire Hess diagram

$$
\log \mathcal{L}=\sum_{i} \log \mathcal{P}\left(k_{i} ; \mu_{i}\right)
$$

In practice, we assign a low (non-zero) probability of having a star even in pixels where $\mu_{i}=0$. This is essentially equivalent to allowing a constant "background level" of stars that are not fitted by any isochrone. This is necessary since any star in a pixel with $\mu_{i}=0$ would otherwise immediately drive the total likelihood to zero. Since no combination of existing isochrones provides a perfect match to the observed CMDs, and one may in any case always expect some contamination of the CMD by foreground stars, background galaxies, etc., this would be overly restrictive. We also experimented with other criteria, e.g., minimum rms difference between observed and model Hess diagrams, but tests similar to those described in Sect. 5.2 below indicated that the maximum-likelihood method gave the most reliable results.

To create the synthetic CMD, the program uses theoretical isochrones either from Padova (Marigo et al. 2008) or Geneva (Lejeune \& Schaerer 2001) or any other set of isochrones, as long as they tabulate the relevant color versus mass. If the weight of each isochrone were fitted independently, this would lead to a very large number of free parameters, due to the small difference in age between individual isochrones, e.g., $\Delta \log \tau=0.05$ for the Padova isochrones. We therefore group the isochrones together in age bins that can be defined by the user, typically using a bin size of $\Delta \log \tau=0.15$ dex.

The program allows us to assign different weights to different rectangular regions of the CMD, selected by the user. This is useful because some phases of stellar evolution are more uncertain than others, e.g., the blue loop stars, and thus should carry less weight in the overall determination of the SFH. The use of completeness limits (if known) can be applied before the creation of the synthetic models, interpolating the completeness limits over the whole area of the CMD. The option of applying 
the completeness correction after the creation of the synthetic model is simply a multiplication of each pixel in the synthetic CMD with a number between 0 and 1 . We also include a simplified treatment of binaries, in which binary evolution is ignored but the effect of unresolved binaries on the CMD are modeled. The program currently allows three different assumptions about the mass ratios in binary systems, namely a delta function, an IMF sampling and a flat distribution. The metallicity, (a range of) extinction values, and distance modulus must also be specified. We allow the extinction to be age-dependent, by providing a list of ages and a range of extinction values for each age. The program then interpolates in this list for each isochrone.

Isochrones are populated by assuming a Salpeter (1955) IMF with SFHs normalised to a mass range specify by the user ( $M=[0.15,100] M_{\odot}$ is the default range). Since we use Hess diagrams, each point in the CMD is a density function. To construct them, different kernels, namely square, Gaussian, disc, or delta function, can be used. The kernels are of adjustable resolution and dimension. In the test presented below, we adopted a resolution for the Hess diagrams of $100 \times 100$ pixels and used a delta function kernel. Each isochrone is broadened by the assumed photometric errors and binarity, and shifted and broadened by the (range of) specified extinction values. To ensure a smooth coverage of the CMD, the program interpolates the isochrones by a factor of 10 . After creating of the Hess diagram for each individual isochrone, the program linearly combines them and a synthetic CMD Hess diagram is obtained.

We performed a number of tests to check the internal consistency of the program, as well as the sensitivity of the derived SFHs to the various parameters involved.

\subsection{Internal consistency check of the code}

We created artificial stellar populations, passed them to the program, and then checked how the output compared to the input. The populations were constructed assuming a constant SFR of $0.1 M_{\odot} \mathrm{yr}^{-1}$, Padova or Geneva theoretical isochrones, a Salpeter's IMF, and solar metallicity. The mass of each star was randomly chosen using Salpeter's prescription for masses between 1 and $100 M_{\odot}$ (low-mass stars $\left(\leq 1 M_{\odot}\right)$ are too faint to appear in the Hess diagram, especially after applying a completeness limit). Because we restricted the mass range in our analysis, we then extrapolated to the default mass range used by the code $\left(M=[0.15,100] M_{\odot}\right)$. The age of each star was assigned randomly (from a uniform distribution) for ages between 4 Myr to 1 Gyr. Having the mass and the age of each star, magnitudes were obtained by interpolating the theoretical isochrones. The magnitude limit used in these tests was $M_{V}=0$. We did not include binaries or extinction in this initial internal consistency check of the code.

Figure 10 presents the results of this analysis. In all the panels, the input SFH is shown as a horizontal straight line and the average reconstructed SFH of the output after 10 different runs as dashed lines. Each run had a new population, i.e., different random realization, for the bands $B, V$, and $I$. The error bars illustrate the standard deviation in the reconstructed SFHs. We studied the variations in these results based on the different assumptions of binning. Three different bins were used in this test corresponding to each row of Fig. 10.

We see that the reconstructed star formation histories generally agree fairly well with the input values regardless of the binning, color, or isochrones used. It is observed that the error bars are larger for smaller bins, although good agreement with the input $\mathrm{SFH}$ is maintained. The maximum difference is $\Delta \log (\mathrm{SFR}) \sim 0.05 \mathrm{dex}$, and generally far less.

We performed a test to see how completeness affects our SFH estimation. Figure 11 shows the Hess diagram of a stellar population with ages between $10^{6}$ to $10^{9} \mathrm{yr}$ using the mass range from above. Overplotted are 3 Padova 2008 isochrones for ages $10^{7}, 10^{8}$, and $10^{9} \mathrm{yr}$ and four completeness limits at $M_{V}=-0.5,0.0,0.5$, and $1.0 \mathrm{mag}$. Figure 12 shows that the SFHs can be recovered reliably to progressively older ages as the completeness limit becomes fainter. In particular, the "burst" at $10^{8.8}$ years disappears when fainter stars are included in the fit.

Cignoni \& Tosi (2010) created artificial CMDs to simulate a stellar population with a constant SFR between 0-13 Gyr and used four different completeness limits. They concluded that to safely reconstruct a SFH over a full Hubble time from a CMD, we need to resolve all stars down to $M_{V}=4.5$. However, this completeness limit can only be reached for galaxies in the Local Group.

We conclude that our program produces consistent results at the level of $0.05 \mathrm{dex}$, the age range over which this holds being restricted mainly by the completeness limits. This internal consistency of the code does not, however, imply that real SFHs can be recovered with similar accuracy.

\subsection{Tests of sensitivity to assumptions about parameters}

We now concentrate on testing the effects of four parameters: metallicity, extinction, binarity, and the fitting area used (rectangular boxes). To perform this test, we executed the code using the scientific data described in Sect. 4, using different assumptions about these parameters and a mass range $M=[0.10,100] M_{\odot}$ (which is the same mass range used in Sect. 5.4). The results are shown in Fig. 13. Each panel represents one of the tested variables.

In panel $a$ ), we show the SFHs derived for three assumptions about metallicity: solar $(Z=0.02)$, LMC $(Z=0.008)$, and SMC $(Z=0.004)$. In this panel, we ignore binaries, include no additional extinction and used the fitting area called "boxes2" described below. We observed that the SFH does not change very much from LMC to solar, but at SMC metallicity there is a much stronger increase from $\sim 100 \mathrm{Myr}$ ago to the present. By comparing the observed and model Hess diagrams, it is clear that the solar metallicity isochrones generally provide a poor fit, especially for the red and blue supergiants that appear much too cool compared to the observations, while LMC and SMC metallicities are very similar (see Fig. 14).

Panel $b$ ) presents the behavior of the SFHs for three different assumptions about the total extinction (foreground plus internal): $A_{b}=[0.1,0.15,0.2]$. We fixed the metallicity at $Z=0.008$ (LMC), again ignoring binaries, used Padova isochrones and used the fitting area "boxes2". In general, the SFHs obtained using different extinctions do not vary strongly for these relatively modest variations in the extinction. Greater extinction variations would produce larger shifts towards the red in the model Hess diagrams and are inconsistent with the data.

Panel $c$ ) shows the dependence on the assumptions for binary fraction $(f)$ and mass ratio $(q)$ of values (1.) $f=0, q=0$; (2.) $f=0.5, q=[0.1,0.9]$; (3.) $f=1, q=1$. The panel also indicates how this dependence affects the estimation of the SFHs. We used Padova isochrones, fixed the metallicity at $Z=0.008$, ignored the effects of additional extinction, and used the fitting area named "boxes2" described below. Binarity can have an effect on the derived SFHs with minor effects for $\tau \leq 100 \mathrm{Myr}$ and 


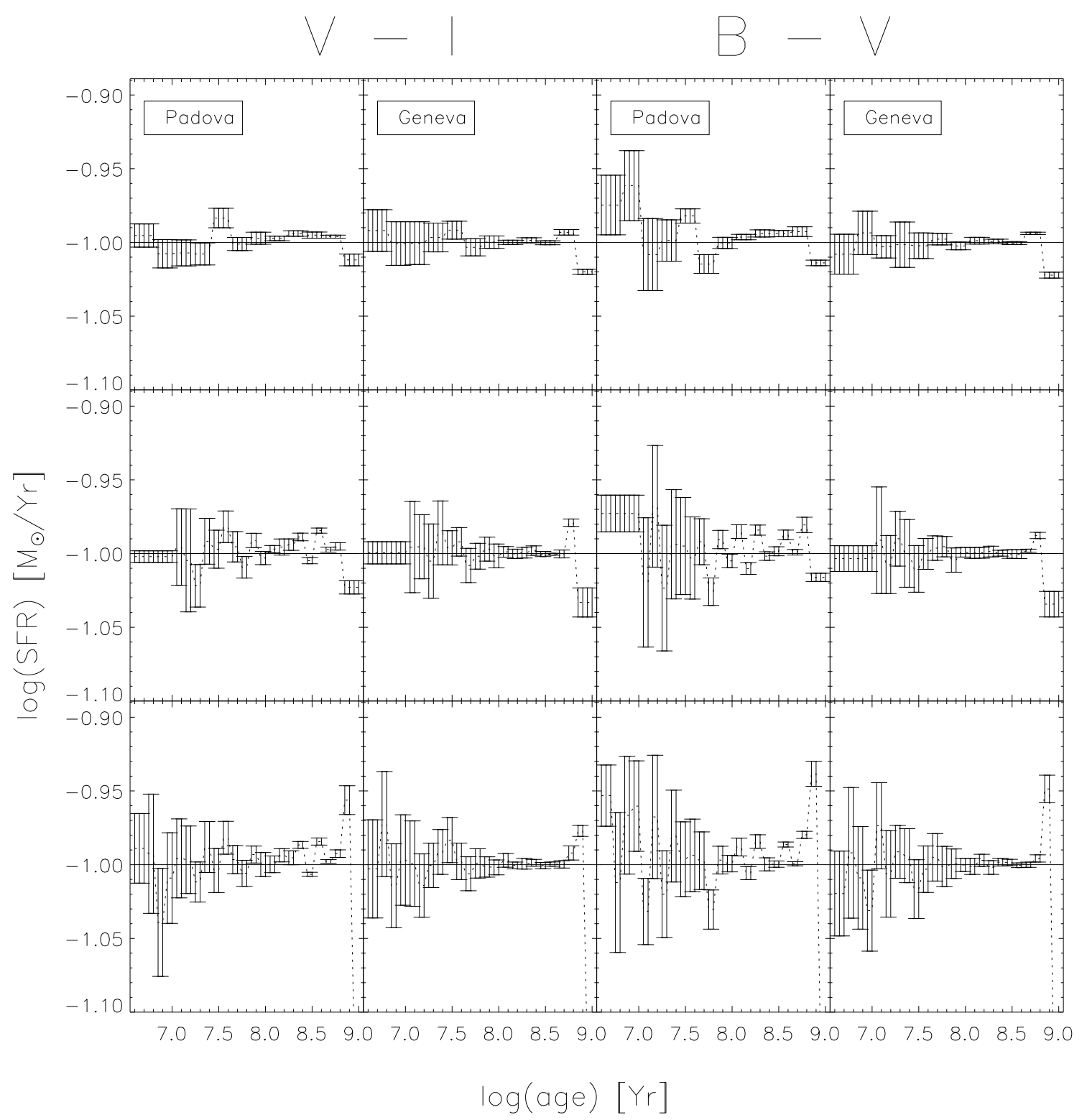

Fig. 10. SFH test using the bands $B, V$, and $I$. Artificial populations were created using Padova and Geneva isochrones and then passed to the program. The procedure was performed 10 times and the mean SFH (dashed line) and the respective standard deviation is shown, relative to the input assumed SFH of $0.1 M_{\text {sun }} \mathrm{yr}^{-1}$ (straight line). Each row represents a different binning used for the study, which decreases in size from top to bottom.

a shift towards somewhat lower overall SFR for the extreme case $f$ and $q$ equal 1 . However, the binarity assumptions made here are not completely realistic. The first and third assumptions are extreme cases with no binaries at all and a galaxy where all the stars have a companion of exactly the same mass, respectively. The second assumption is an intermediate case where there is a continuous (flat) range of $q$ values and a star has $\sim 50 \%$ probability of being in a binary system. However, in a more realistic case binary evolution should be taken into account as well. This is however beyond the scope of the present work.

In the last panel, panel $d$ ), we show the SFH estimates obtained using two different sets of boxes in the fitting. As overplotted in Fig. 17, where we plot the observed Hess diagram and the regions fitted, the left box $(-0.5 \leq V-I \leq-0.05$ and $-8.0 \leq V \leq-2.5)$ used contains the main sequence stars, the blue He core burning phases being outside of the fit, while the right box $(1.0 \leq V-I \leq 2.5$ and $-8.0 \leq V \leq-2.0)$ contains the red He core burning stars and possible RGBs and AGBs. This set of boxes is called "boxes2". The second set of boxes covers the same area for the evolved stars (right box), while the blue He core burning stars are also included in the fitting box $(-0.5 \leq V-I \leq 0.7)$, which we label "boxes1" in panel $d)$. In general, we found that, the SFH result from "boxes2" shows a lower value that for "boxes1". We studied these two possible sets of boxes because the fitted Hess diagrams retrieved using these two boxes indicated that the fit for the blue He core burning stars is not in good agreement with observations. The variations after $\tau \sim 10^{8.7} \mathrm{yr}$ are unlikely to be real, but are probably due to incompleteness as discussed above.

We created a fake population using Padova isochrones and assuming solar metallicity, no extinction, and no binarity; we then analyze this with our program, using Geneva isochrones to see whether the assumed SFH could be recovered. Figure 16 shows the result of this test. There are significant differences 


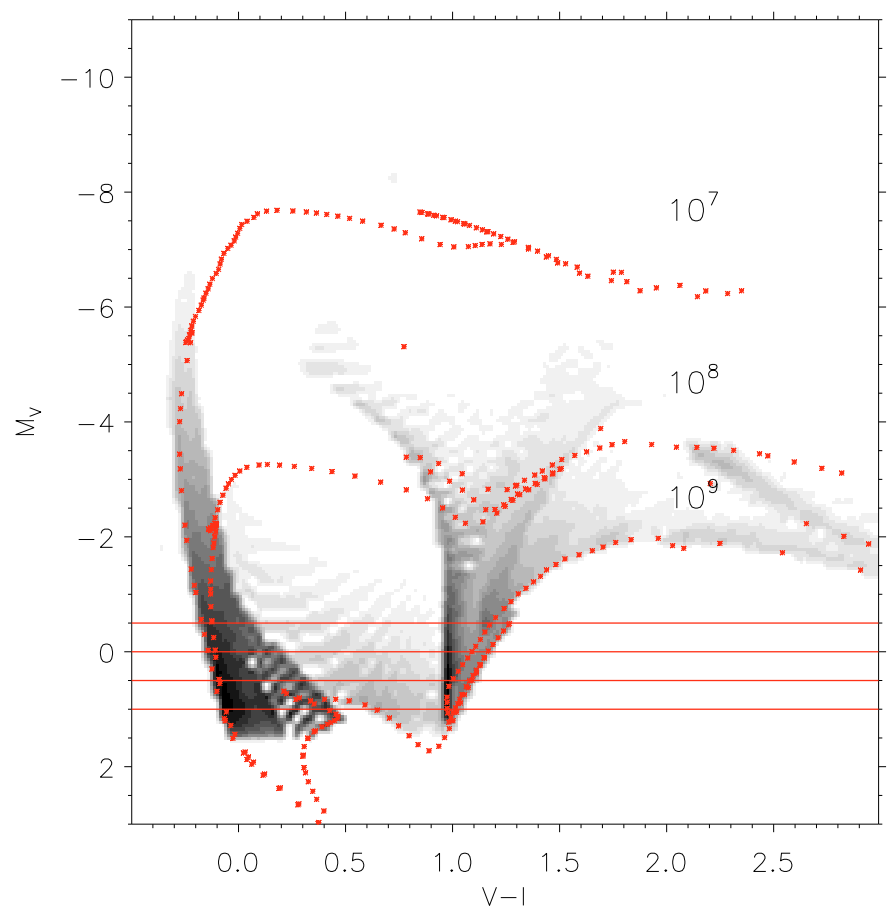

Fig. 11. Hess diagram for the 4 possible completeness limit studied in Fig. 12.

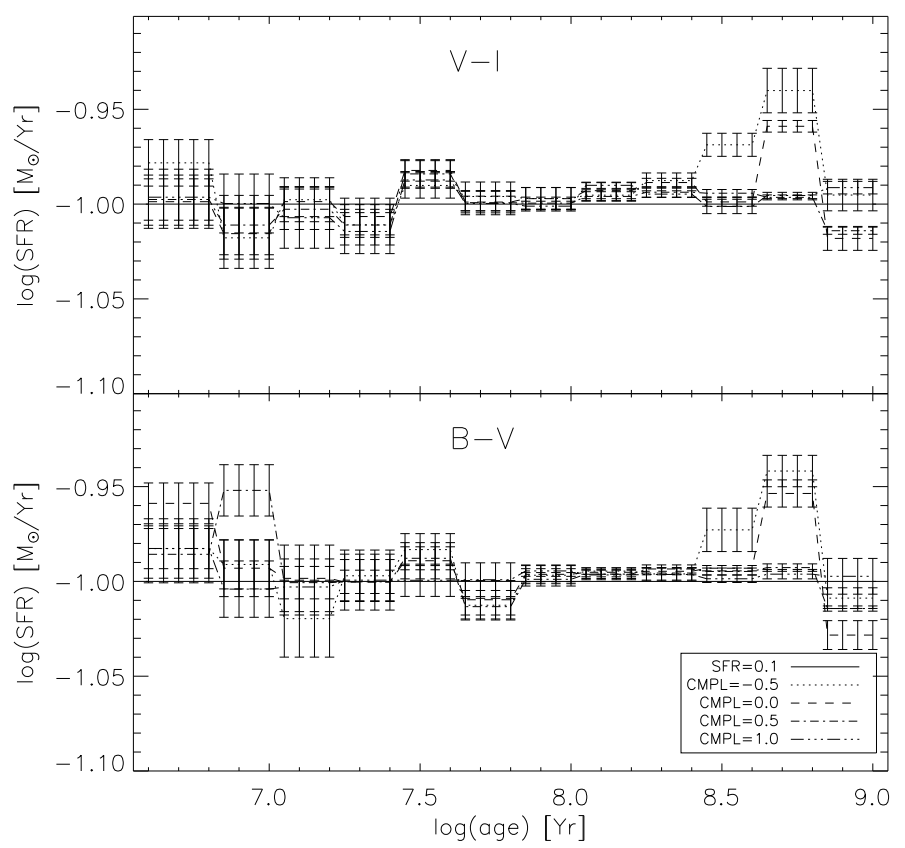

Fig. 12. Test of the SFH using different completeness limits.

between the input and recovered $\mathrm{SFH}$, deviations being as large as $\sim 0.4$ dex at young ages $(<10 \mathrm{Myr})$ and differences being at the level of $0.1-0.2$ dex at older ages. The error bars presented in Fig. 16 were determined using the same method used in the previous section, i.e., are equivalent to the standard deviation of the reconstructed SFHs after 10 different runs with different random realizations.

We finally test how random errors (due to the finite number of stars in the Hess diagram) affect the SFHs, using a bootstrapping method whereby the input data, i.e., the photometric data found in this work, are randomly resampled many times.
To perform this test, we used the observations obtained in this work (see Sect. 4), Padova 2008 isochrones, LMC metallicity, no additional extinction, and no binarity. Disabling binarity will not influence our results dramatically and will save us computational time. We ran the program 100 times, redistributing randomly the photometric data in each run. From Fig. 15, we conclude that Poisson variations do not significantly affect our results.

To summarize, we demonstrated that the main uncertainties in our derived SFHs are systematic, depending on assumptions about binarity, the choice of isochrones, and metallicity. However, with the exception of the use of solar metallicity isochrones (which provide a poor fit to the data), the overall mean SFRs are fairly consistent with our data up to ages of a few hundred Myr.

\subsection{Results}

To infer the SFH of NGC 4395, we combined both fields to be able to directly compare with the cluster age distribution. The differences in our completeness limits for each band and each field are smaller than $0.1 \mathrm{mag}$, allowing us to use the completeness values obtained in our first field (results for the second field can also be used instead) to study the SFH of the whole galaxy. In the SFH reconstruction, we used a resolution of $200 \times 200$ pixels for the Hess diagrams and a Gaussian kernel with a standard deviation of $0.02 \mathrm{mag}$ in $V-I$ (or $B-V$ ). Based on our observed Hess diagram we used 2 rectangular fitting areas (boxes 2 ) for the SFH reconstruction with the same limits as described in the previous section. To check the consistency of our results we performed the analysis using both $B-V$ and $V-I$ colors, using the same parameters and boxes to fit the data.

Figure 17 depicts the observed (left) and best-fit model (right) Hess diagrams (assuming $f=0.5$ and $q=[0.1,0.9]$ ). The final combination of parameters used to estimate the SFH were Padova 2008 isochrones, a Salpeter IMF $\left(\mathrm{M}=[0.10,100] M_{\odot}\right)$, LMC metallicity, three different assumptions for the binarity, distance modulus of 28.1, a total extinction of $A_{B}=0.15$, and the photometric errors and completeness limit obtained in Sect. 4.

The phases that are most accurately reproduced by the fit are the main sequence, red core-He burning, and RGB/AGB phases. For the blue core-He burning phases, the model fit is less good, being redder than observed and with a more pronounced gap between the main sequence and blue core-He burning stars in the model than in the data. This is a generic problem with the isochrones that exists for any star formation history we adopt.

Our most accurate estimates of the extinction were obtained by trial and error. We compared the main sequence stars from the fitted Hess diagram with data for those observed and assumed different values $\left(A_{B}=[0.1,0.15,0.2]\right)$ for the total extinction until we obtained the best fit between the two Hess diagrams (considering the main sequence only). The difference between the foreground value $\left(A_{B}=0.074\right)$ and our best-fit model value $\left(A_{B} \sim 0.15\right)$ suggests a low internal extinction in NGC 4395 of $A_{B} \approx 0.076 \mathrm{mag}$. This estimation was performed for LMC metallicity.

The estimated SFHs (for $V-I$ and $B-V$ ) are shown in Fig. 18. As discussed in the previous section, the SFHs become very uncertain at ages greater than a few hundred Myr; the apparent burst at $\sim 10^{8.4} \mathrm{yr}$ is probably not real. There is also a hint of a rapid drop at very young ages in $V-I\left(\sim 10^{6.8}\right.$ years $)$, but this may be caused by uncertainties in the isochrones. Furthermore, the youngest age included in our artificial Hess diagrams is $4 \mathrm{Myr}$; if younger stars were present in the field, these would be included in the youngest bin. The appropriate lower age limit depends 


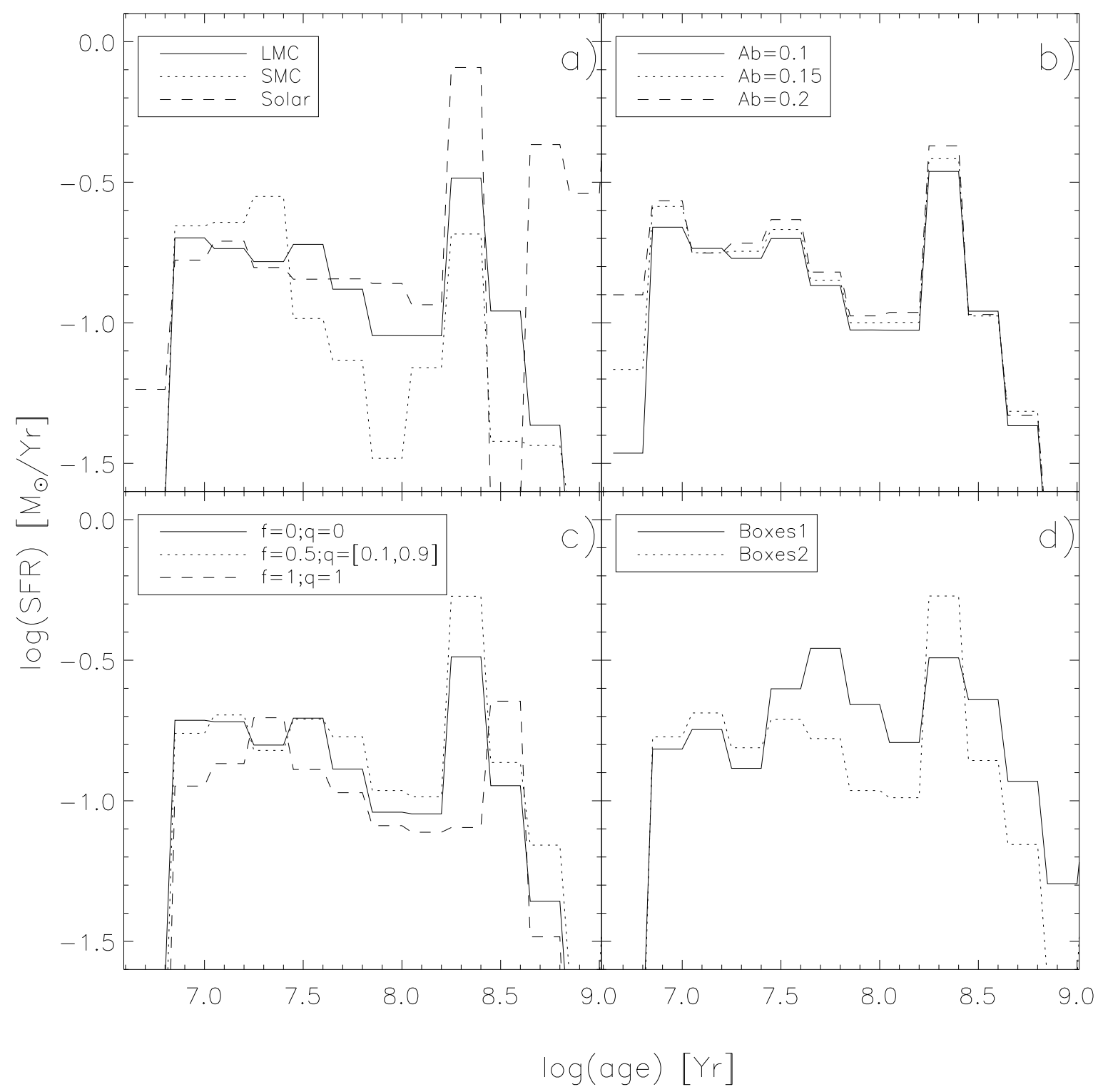

Fig. 13. Padova theoretical isochrones were used to test the SFH program developed using the photometric data obtained in Sect. 4. Panel a): test for different metallicities (solar, LMC, SMC) without binarity or extinction assumed. Panel b): test for different extinctions assuming LMC metallicity and no binarity. Panel c): test for different binarity assuming LMC metallicity and no extinction. Panel d): test for different boxes used over the fitting data assuming LMC metallicity, no extinction, and no binarity.

on how long young stars are embedded in their native molecular clouds. We present the SFHs for different binarity assumptions because this parameter is also uncertain. We use the same 3 choices in Fig. 18 as those used in Fig. 13 above. Taking the values for each bin (in linear units), we estimated the average star formation rate for ages between $10^{7}$ and $10^{8}$ years using the colors $V-I$ and $B-V$, as shown in Table 3 .

There is an apparent increase in the SFR between $\log \tau \sim$ $10^{8.0}$ and $\log \tau \sim 10^{7.0}$, of $\sim 0.5$ dex. Based in Fig. 18 , the average star formation rate over the past $10^{8}$ years is SFR $\approx 0.17 M_{\odot} \mathrm{yr}^{-1}$ within the two ACS fields (assuming $f=0.5$ and $q=[0.1,0.9]$ ). We note that this is a factor of 4 higher than the global SFR for NGC 4395 that follows from using the far-infrared luminosity described in Larsen \& Richtler (2000), who used the calibration by Buat \& Xu (1996). This highlights the difficulty in determining extragalactic star formation rates from integrated light.

\subsection{Ages and masses of clusters}

After identifying the star cluster candidates (see Sect. 4) in the two fields, we need to estimate their masses and ages. These parameters were estimated using the program AnalySED, created by Anders et al. (2004). This program determines the masses and ages using GALEV SSP evolutionary models (Schulz et al. 2002). The parameters used for AnalySED are a Salpeter IMF (Salpeter 1955) in the mass range 0.10 to $100 M_{\odot}$, Padova isochrones (Girardi et al. 2000), and a LMC metallicity. AnalySED performs a match, comparing the observed and theoretical spectral energy distributions (SEDs), based on GALEV SSP models. As output, AnalySED provides, among other parameters, an estimation of the mass and the age for each cluster.

In addition to our magnitude limit of $V=23\left(M_{V} \sim-5\right)$ for our clusters, we also define a lower mass limit at $10^{2.5} M_{\odot}$ to increase the likelihood that extended objects are true clusters 


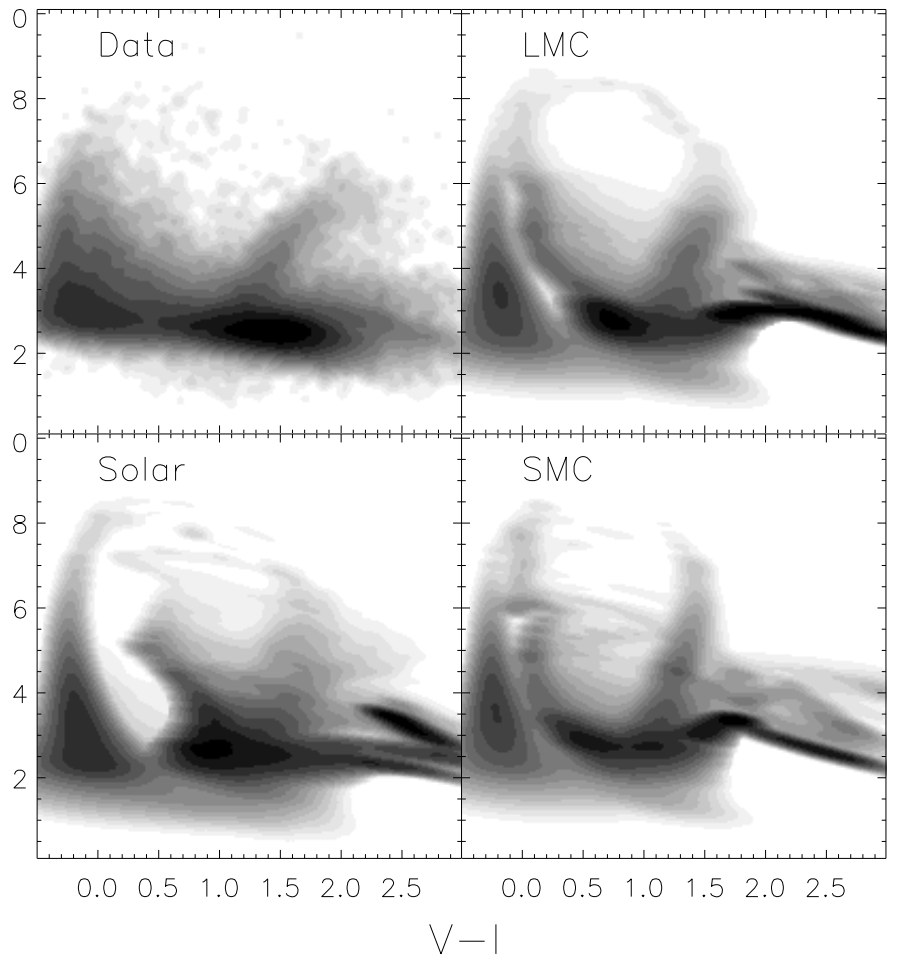

Fig. 14. Comparison of the fit for different metallicities (solar, LMC, and $\mathrm{SMC}$ ).

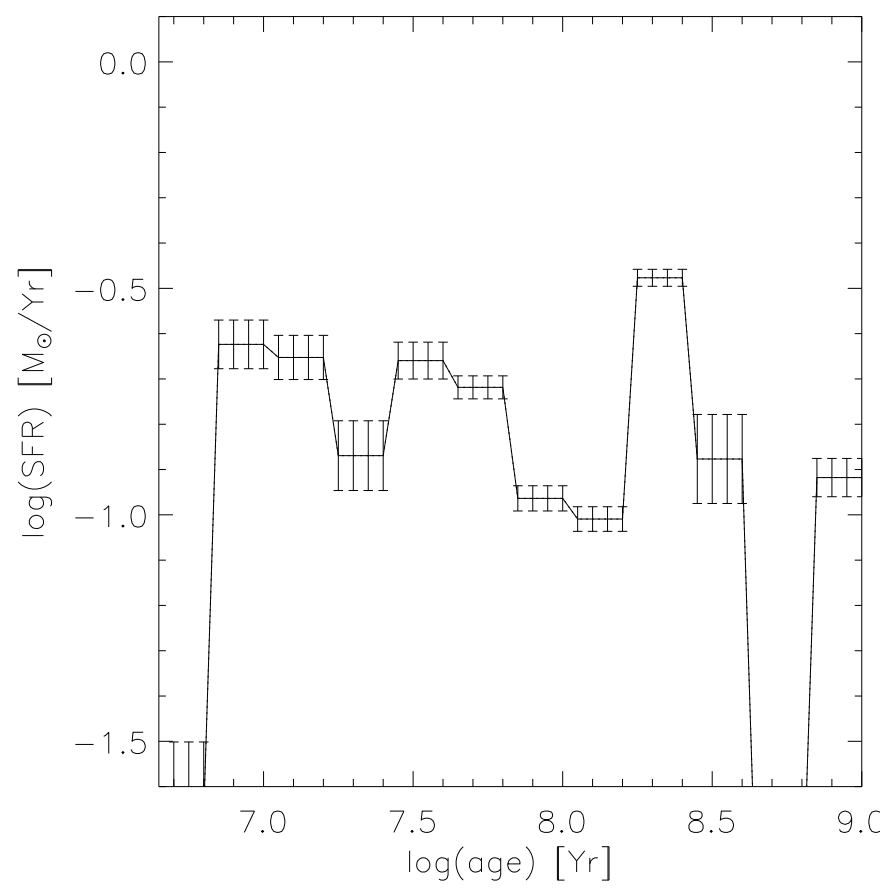

Fig. 15. Bootstrapping study. The errors retrieved from this test are the random errors of our program. No strong variations caused by random errors affect our results.

and not just chance projections of a few bright stars. However, over most of the age range $(\log ($ age $) \geq 7)$, these false detections would fall below our magnitude limit.

Figure 19 shows the age-mass diagram of the cluster candidates in NGC 4395. The number of clusters detected is rather small because the two HST pointings do not cover the whole

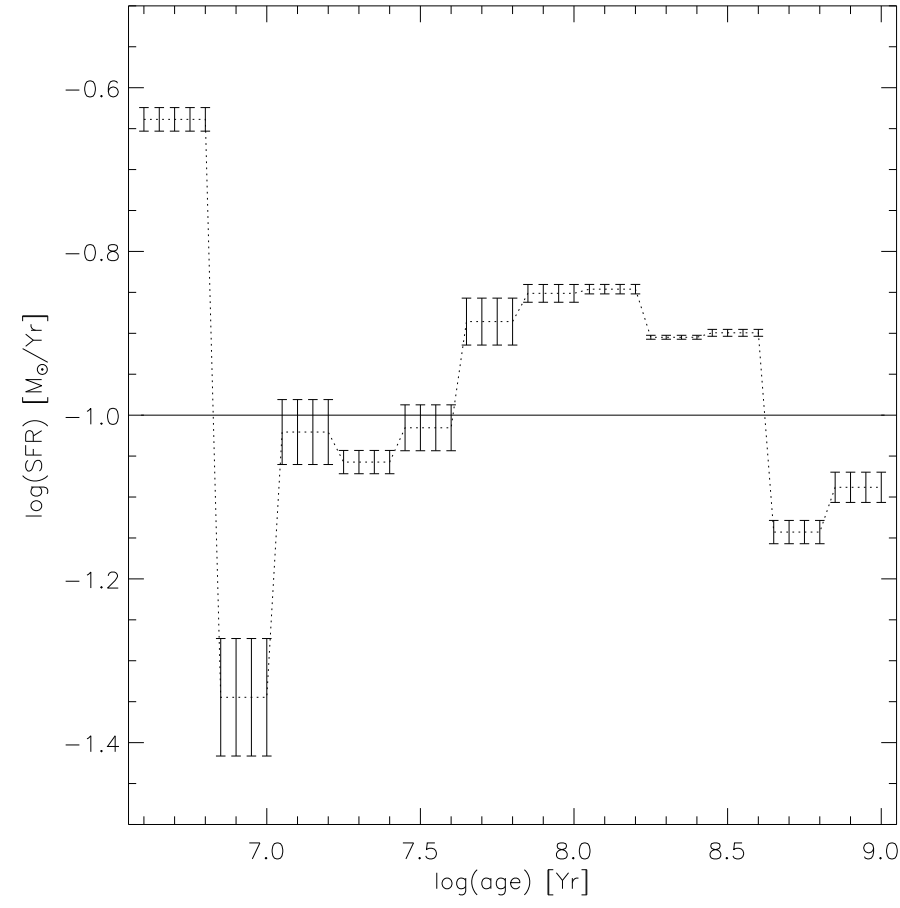

Fig. 16. Test using Padova isochrones to create the population and Geneva isochrones to analyze them.

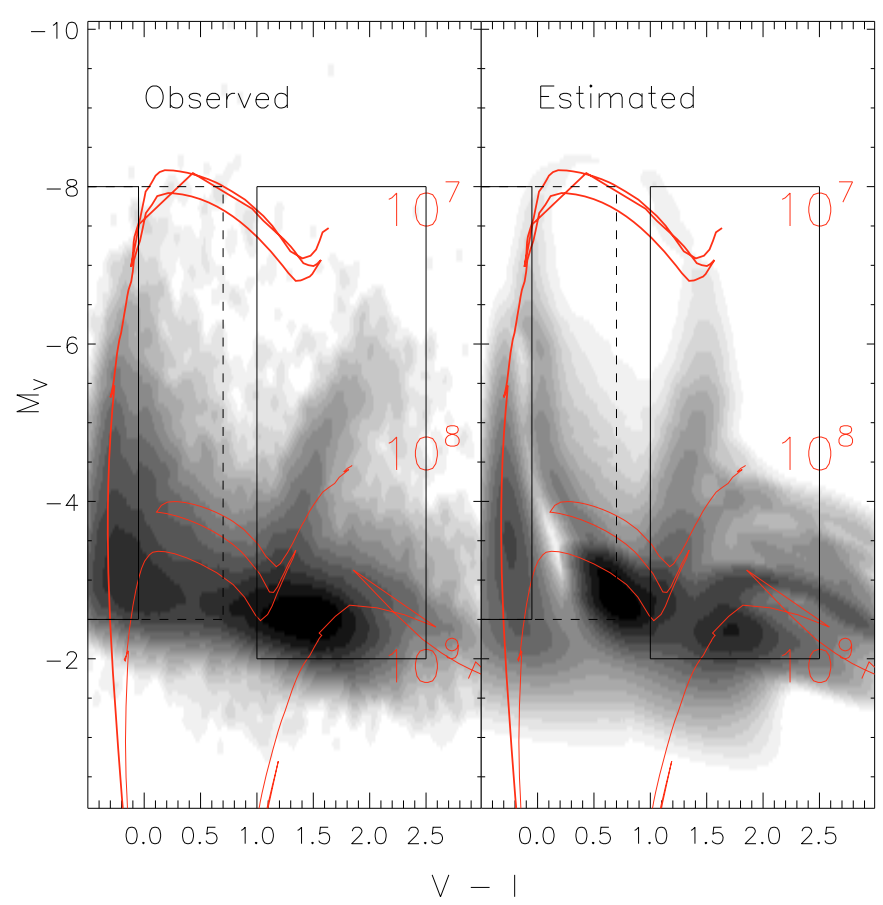

Fig. 17. Comparison between the observed and fitted Hess diagrams for the field stars studied in this work. As age indicators, three isochrones are overplotted at the ages of $10^{7}, 10^{8}$, and $10^{9} \mathrm{yr}$. Boxes used to fit the data are represented for both Hess diagrams, observed and fitted.

galaxy and our coverage for clusters is reduced further by our requirement of the WFPC 2 pointings for age-dating. We see that neither massive $\left(M \geq 10^{5} M_{\odot}\right)$ nor old $\left(\tau \geq 10^{9}\right.$ yr) clusters are detected, in agreement with previous work (Larsen \& Richtler 1999; Mora et al. 2009). We identified few clusters with $\tau>100$ Myr for LMC metallicity in agreement with Mora et al. (2009), although the number of objects was greater in their work. 


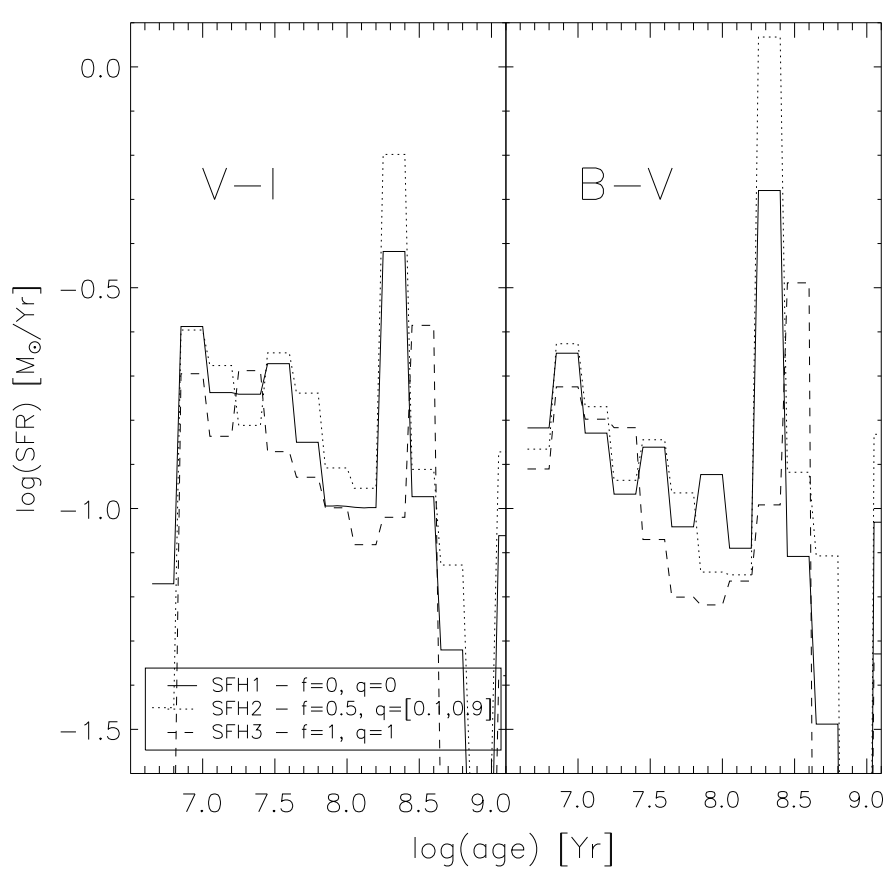

Fig. 18. SFH for the galaxy NGC 4395. We studied the SFH for three different assumptions about the binaries trying to cover possible combinations between binary fraction and mass ratio for the colors $V-I$ (left panel) and $B-V$ (right panel).

Table 3. Average star formation rate [in $M_{\odot} \mathrm{Yr}^{-1}$ ] over the past $10^{8}$ years, estimated using different binarity, colors, and isochrones.

\begin{tabular}{cccc}
\hline \hline & $f=0$ & $f=0.5$ & $f=1$ \\
\hline & $q=0$ & $q=[0.1,0.9]$ & $q=1$ \\
& & Padova & \\
$V-I$ & 0.18 & 0.19 & 0.15 \\
$B-V$ & 0.14 & 0.14 & 0.12 \\
& & Geneva & \\
$V-I$ & 0.20 & 0.21 & 0.19 \\
$B-V$ & 0.16 & 0.20 & 0.16 \\
\hline
\end{tabular}

Nevertheless, the number of objects detected at these ages (and greater) varies, as can be see in Fig. 7 of Mora et al., depending on the metallicity used to estimate the parameters.

\section{Summary, discussion, and conclusions}

We have described our procedures to obtain the age distributions of field stars and star clusters in HST images of nearby $(D \approx 4$ $\mathrm{Mpc}$ ) galaxies, in particular, we have described our implementation of the synthetic CMD method and tests of our program developed for this purpose. We found that the code recovers the star formation histories of synthetic populations with good accuracy, while errors in the derived SFHs of true populations are dominated by systematics errors. We have derived the SFH for NGC 4395 for three different metallicities and find an approximately constant SFR over the past few hundred Myr assuming LMC-like metallicity. Only a modest amount of internal extinction $\left(A_{B} \approx 0.08\right)$ in NGC 4395 is required in addition to the Galactic foreground extinction to match the data. Hence, uncertainties in the total extinction is not a major source of error compared to, for example, binary stars and the choice of isochrones. Different assumptions may lead to changes in our estimated SFRs of up to a factor of $2-4$ in specific age bins,

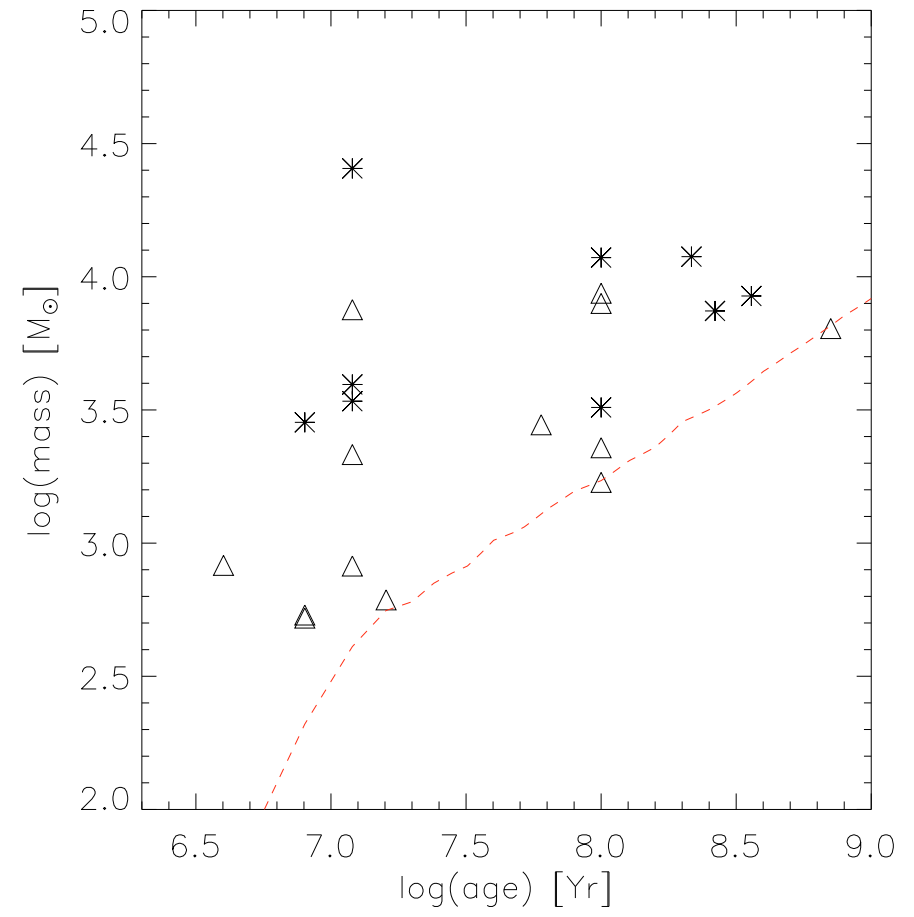

Fig. 19. Age-mass distribution of the clusters detected in our two fields observed. The red dashed line represents $M_{V}=-5$. Symbols are the same as in Fig. 6.

although the global average will be less affected. Poissonian errors do not contribute substantially to the uncertainties because of the large number of field stars used in our analysis.

After estimating the SFH of the field stars and both the age and mass distributions of star clusters, we can now measure which fraction of stars are in clusters. We compare the fractions for cluster ages between $10^{7}$ and $10^{8}$ years, since disruption due to tidal shocks (GMC and spiral arm encounters) and evaporation is expected to become significant at older ages, while younger objects may still be unbound and prone to "infant mortality".

A rough estimate of the cluster formation rate (CFR) can be obtained by dividing the total amount of mass in clusters observed by the age range. From $10^{7} \leq \tau \leq 10^{8} \mathrm{yr}$, the total mass observed in Fig. 19 is $M_{\text {obs }} \sim 8.3 \times 10^{4} M_{\odot}$, which infers a $\log (\mathrm{CFR}) \approx-3.03\left[M_{\odot} \mathrm{yr}^{-1}\right]$. We correct this for the smaller area covered by the WFPC2 camera compared to the ACS (a factor of 2.27$)$ and thus obtain $\log (\mathrm{CFR}) \approx-2.68\left[M_{\odot} \mathrm{yr}^{-1}\right]$ for the same equivalent area covered by our field star data.

A magnitude-limited sample does not allow us to observe all the clusters in the age range. Using the observed total mass and assuming a cluster mass function $(\Psi(m))$, we can estimate the total mass in clusters for a certain age range. Larsen (2009) showed that a Schechter function (with $M_{\star}=2 \times 10^{5} M_{\odot}$ ) can model the initial cluster mass function in present-day spiral discs. However, the canonical cluster mass function, a power law with index -2 , can be used for the same purpose. To estimate the cluster formation rate we used both functions and calculated the total mass in the age range mentioned above. Since the most massive clusters observed have $M<10^{5} M_{\odot}$, it makes little difference whether we adopt the Schechter function or an untruncated power-law. 


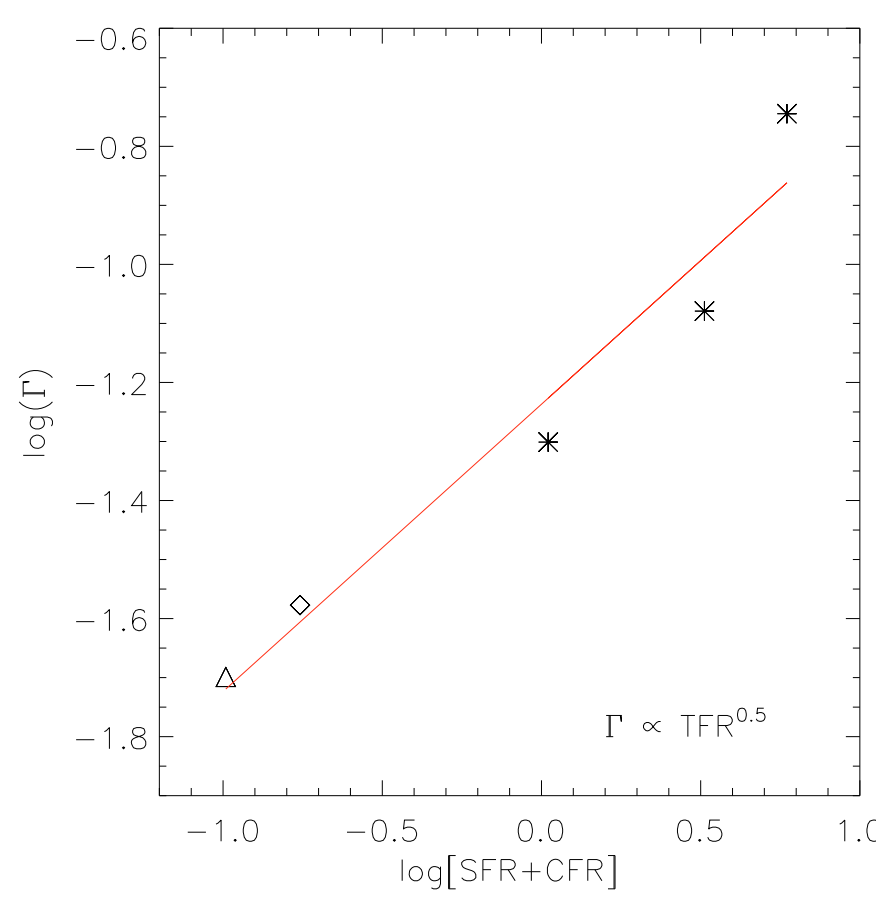

Fig. 20. Relation between the cluster formation efficiency $(\Gamma)$ and the total formation rate (TFR $=\mathrm{SFR}+\mathrm{CFR})$. An apparent power law function fits the date following the equation $\Gamma \approx \mathrm{TFR}^{0.5}$. Symbols represent: $\star$ are data from Gieles (2009) for the galaxies M 74, M 101 and M 51; $\triangle$ is for the SMC (Gieles \& Bastian 2008); and $\diamond$ our estimation for NGC 4395.

The total mass in the cluster system was estimated using the equation

$M_{\mathrm{tot}}=M_{\mathrm{obs}} \times \frac{\left(t_{2}-t_{1}\right) \int_{m_{\mathrm{lo}}}^{m_{\mathrm{up}}} m \Psi(m) d m}{\int_{t_{1}}^{t_{2}} \int_{m_{\mathrm{lim}(t)}}^{m_{\mathrm{up}}} m \Psi(m) \mathrm{d} m \mathrm{~d} t}$,

where $t_{1}, t_{2}$ represent the time interval $\left(t_{1}=10^{7}\right.$ years, $t_{2}=$ $10^{8}$ years), $m_{\mathrm{lo}}$ and $m_{\text {up }}$ are the lower and upper mass limits over which the mass is normalized $\left(m_{\mathrm{lo}}=10\right.$ and $\left.m_{\mathrm{up}}=10^{6} M_{\odot}\right)$, and $m_{\lim (t)}$ is the (age-dependent) detection limit of our cluster sample (dashed line in Fig. 19). Since the last parameter, $m_{\text {lim }}$, is derived from the SSP models used, this integral must be evaluated numerically. Equation (2) assumes a uniform cluster age distribution, which is the most conservative choice we can make given the small number of clusters in our sample and the uncertainties in the age determinations (Fig. 6). We also assume that $\Psi(m)$ is age-independent, meaning that we ignore disruption. Applying the correction for undetected clusters, we obtained values for the CFR of $\log (\mathrm{CFR})=-2.37$ and $\log (\mathrm{CFR})=-2.35$ for power law and Schechter functions respectively, values that are corrected for the area covered by the HST detectors mentioned above. We have also tried applying Eq. (2) to 3 sub-bins in age, which changed the total overall CFR by a very small amount. We note that, strictly speaking, we are not measuring the true cluster formation rate, but rather the current age distribution of surviving clusters. Dividing these numbers by the SFR $\left(\mathrm{SFR}=0.17 M_{\odot} \mathrm{yr}^{-1}\right)$, we obtain a ratio of $\Gamma=\mathrm{CFR} / \mathrm{SFR}=0.0263$.

Our estimate of $\Gamma$ is lower than the value $\Gamma \sim 0.08$ obtained by Bastian (2008). Gieles (2009) estimated $\Gamma$ for three galaxies namely M74, M 101, and M 51. The cluster formation rate was estimated by comparing theoretical with empirical luminosity functions. Gieles suggested that $\Gamma$ has the tendency to increase with SFR. Gieles \& Bastian (2008) estimated $\Gamma \approx 0.02$ for the SMC using an accurate determination of the mean global star formation rate based on the field stars $\left(\mathrm{SFR} \approx 0.1 M_{\odot} \mathrm{yr}^{-1}\right.$ ) performed by Harris \& Zaritsky (2004) and the CFR calculated in their work. Other studies have found the same value for $\Gamma$ in the SMC (see Goddard et al. 2010). We took the data from Gieles (2009) (see Table 1 in his article), and Gieles \& Bastian (2008) and plotted the total formation rate (TFR $=$ SFR + CFR) against $\Gamma$ for these four galaxies and included our CFR and SFR for NGC 4395 to attempt to detect any correlation. Figure 20 shows the plot of $\log (\Gamma)$ versus $\log (\mathrm{TFR})$. There is an apparent correlation between these two values, which can be approximated by a power law $\Gamma \propto \mathrm{TFR}^{0.5}$. Although only five points are plotted and the TFR in NGC 4395 does not cover the whole galaxy, Fig. 20 does suggest that the relatively low $\Gamma$ value we derive for NGC 4395 is consistent with a general trend. In a future study (Silva-Villa et al. 2010, in prep.), we will include more points in this plot for galaxies spanning a range in TFR and check the validity of this result.

Goddard et al. (2010) found an empirical relation between $\Gamma$ and the SFR density $\left(\Sigma_{\mathrm{SFR}}\left[M_{\odot} \mathrm{yr}^{-1} \mathrm{Kpc}^{-2}\right]\right)$. We estimated $\Sigma_{\mathrm{SFR}}$ to be $\sim 4.65 \times 10^{-3} M_{\odot} \mathrm{yr}^{-1} \mathrm{Kpc}^{-2}$ for NGC 4395 , which leads to $\Gamma \approx 8 \%$ based on the Goddard et al. (2010) relation. This value is higher than that estimated in this work $\Gamma \approx 3 \%$. We point out that the area covered by our observations is not the total area of the galaxy and so the SFR density found in this work might be higher than the global average.

Acknowledgements. Silva-Villa would like to thank Peter Anders for help with the program AnalySED. We thank C. U. Keller and the referee for comments that helped improve this article.

\section{References}

Anders, P., \& Fritze-v. Alvensleben, U. 2003, A\&A, 401, 1063

Anders, P., Bissantz, N., Fritze-v. Alvensleben, U., et al. 2004, MNRAS, 347, 196

Annibali, F., Tosi, M., Monelli, M., et al. 2009, AJ, 138, 169

Aparicio, A., \& Gallart, C. 2004, AJ, 128, 1465

Aparicio, A., \& Hidalgo, S. L. 2009, AJ, 138, 558

Aparicio, A., Gallart, C., \& Bertelli, G. 1997, AJ, 114, 669

Barker, M. K., Sarajedini, A., Geisler, D., Harding, P., \& Schommer, R. 2007, AJ, 133, 1138

Bastian, N. 2008, MNRAS, 390, 759

Bastian, N., \& Gieles, M. 2008, in Mass Loss from Stars and the Evolution of Stellar Clusters, ed. A. de Koter, L. J. Smith, \& L. B. F. M. Waters, ASP Conf. Ser., 388, 353

Baumgardt, H. 2009, Dissolution of Globular Clusters, ed. T. Richtler, \& S. Larsen, 387

Bertelli, G., Bressan, A., Chiosi, C., Fagotto, F., \& Nasi, E. 1994, A\&AS, 106, 275

Bertin, E., \& Arnouts, S. 1996, A\&AS, 117, 393

Boutloukos, S. G., \& Lamers, H. J. G. L. M. 2003, MNRAS, 338, 717

Brown, T. M., Beaton, R., Chiba, M., et al. 2008, ApJ, 685, L121

Buat, V., \& Xu, C. 1996, A\&A, 306, 61

Cerviño, M., \& Luridiana, V. 2006, A\&A, 451, 475

Cignoni, M., \& Tosi, M. 2010, Advances in Astronomy, 2010, 3

Cole, A. A., Skillman, E. D., Tolstoy, E., et al. 2007, ApJ, 659, L17

Dolphin, A. 1997, New Astron., 2, 397

Dolphin, A. E. 2000, PASP, 112, 1397

Edvardsson, B., Andersen, J., Gustafsson, B., et al. 1993, A\&A, 275, 101

Elmegreen, B. G. 2008, 388, 249

Fall, S. M. 2006, ApJ, 652, 1129

Filippenko, A. V., \& Sargent, W. L. W. 1989, ApJ, 342, L11

Forbes, D. A., Brodie, J. P., \& Huchra, J. 1997, AJ, 113, 887

Gieles, M. 2009, [arXiv: 0908.2974]

Gieles, M., \& Bastian, N. 2008, A\&A, 482, 165

Girardi, L., Bressan, A., Bertelli, G., et al. 2000, A\&AS, 141, 371

Girardi, L., Chiosi, C., Bertelli, G., et al. 1995, A\&A, 298, 87 
E. Silva-Villa and S. S. Larsen: The star cluster-field star connection in nearby spiral galaxies

Goddard, Q. E., Bastian, N., \& Kennicutt, R. C. 2010, MNRAS, 405, 857

Grevesse, N., \& Sauval, A. J. 1998, Space Sci. Rev., 85, 161

Harris, J., \& Zaritsky, D. 2001, ApJS, 136, 25

Harris, J., \& Zaritsky, D. 2002, in Observed HR Diagrams and Stellar Evolution, ed. T. Lejeune \& J. Fernandes, ASP Conf. Ser., 274, 600

Harris, J., \& Zaritsky, D. 2004, AJ, 127, 1531

Harris, J., \& Zaritsky, D. 2009, AJ, 138, 1243

King, I. 1962, AJ, 67, 471

Koekemoer, A. M., Fruchter, A. S., Hook, R. N., et al. 2002, in The 2002 HST Calibration Workshop : Hubble after the Installation of the ACS and the NICMOS Cooling System, ed. S. Arribas, A. Koekemoer, \& B. Whitmore, 337

Lada, C. J., \& Lada, E. A. 2003, ARA\&A, 41, 57

Lamers, H. J. G. L. M., \& Gieles, M. 2008, in Mass Loss from Stars and the Evolution of Stellar Clusters, ed. A. de Koter, L. J. Smith, \& L. B. F. M. Waters, ASP Conf. Ser., 388, 367

Lamers, H. J. G. L. M., Gieles, M., Bastian, N., et al. 2005, A\&A, 441, 117

Larsen, S. S. 1999, A\&AS, 139, 393

Larsen, S. S. 2009, A\&A, 503, 467

Larsen, S. S., \& Richtler, T. 1999, A\&A, 345, 59

Larsen, S. S., \& Richtler, T. 2000, A\&A, 354, 836

Larsen, S. S., Forbes, D. A., \& Brodie, J. P. 2001, MNRAS, 327, 1116

Larsen, S. S., Efremov, Y. N., Elmegreen, B. G., et al. 2002, ApJ, 567, 896
Larsen, S. S., Mora, M. D., Brodie, J. P., \& Richtler, T 2007, in IAU Symp. 241, ed. A. Vazdekis \& R. F. Peletier, 435

Lejeune, T., \& Schaerer, D. 2001, A\&A, 366, 538

Maíz Apellániz, J. 2009, ApJ, 699, 1938

Marigo, P., Girardi, L., Bressan, A., et al. 2008, A\&A, 482, 883

Mateo, M. L. 1998, ARA\&A, 36, 435

Mora, M. D., Larsen, S. S., \& Kissler-Patig, M. 2007, A\&A, 464, 495

Mora, M. D., Larsen, S. S., Kissler-Patig, M., Brodie, J. P., \& Richtler, T. 2009, A\&A, 501, 949

Rejkuba, M., Greggio, L., \& Zoccali, M. 2004, A\&A, 415, 915

Roy, J., Belley, J., Dutil, Y., et al. 1996, ApJ, 460, 284

Salpeter, E. E. 1955, ApJ, 121, 161

Schlegel, D. J., Finkbeiner, D. P., \& Davis, M. 1998, ApJ, 500, 525

Schulz, J., Fritze-v. Alvensleben, U., Möller, C. S., et al. 2002, A\&A, 392, 1

Sirianni, M., Jee, M. J., Benítez, N., et al. 2005, PASP, 117, 1049

Skillman, E. D., \& Gallart, C. 2002, in Observed HR Diagrams and Stellar

Evolution, ed. T. Lejeune \& J. Fernandes, ASP Conf. Ser., 274, 535

Spitzer, L. 1987, Dynamical evolution of globular clusters, ed. L. Spitzer

Tosi, M., Greggio, L., Marconi, G., et al. 1991, AJ, 102, 951

Whitmore, B. C. 2007, in IAU Symposium, ed. B. G. Elmegreen \& J. Palous, IAU Symposium, 237, 222

Williams, B. F., Dalcanton, J. J., Seth, A. C., et al. 2009, AJ, 137, 419

Young, L. M., Skillman, E. D., Weisz, D. R., et al. 2007, ApJ, 659, 331 